\title{
Common Fixed Point Theorems on Multi-Valued Mappings in 2-Metric Space using T-Hardy Rogers Contraction Condition and F-Contraction
}

\author{
Dinanath Barman ${ }^{1}$, Krishnadhan Sarkar ${ }^{2 *}$ and Kalishankar Tiwary ${ }^{3}$ \\ ${ }^{1,3}$ Department of Mathematics, Raiganj University, Raiganj, West Bengal, \\ India-733134 \\ ${ }^{2}$ Department of Mathematics, Raniganj Girls College, Raniganj, \\ Paschim Bardhaman, West Bengal, India-733158 \\ E-mail: ${ }^{1}$ dinanathbarman85@gmail.com, ${ }^{2 *}$ sarkarkrishnadhan@gmail.com, \\ 3tiwarykalishankar@yahoo.com
}

\begin{abstract}
In this paper we have introduced multi-valued 2-metrices on 2-metric space and have proved some fixed point theorems for multi-valued mappings on 2-metric space using T-Hardy and Rogers type contraction condition. We have also used F-contraction conditions, occasionally weakly compatible to prove the theorems.
\end{abstract}

Keywords: Weakly Compatible, CLRf-property, OWC-property, D-mapping

Mathematics Subject Classification: 47H10, 54H25

\section{INTODUCTION}

IIn 1922, Banach proved a fixed point theorem which is also known as Banach contraction theorem.This theorem has been generalized by various research workers in different spaces for point valued as well as multi valued mappings. It is Nadler (1969) who introduced the notion of multi-valued contraction mapping on metric spaces. Thereafter Joseph and Ramganesh(2013), Abdou(2016) have also worked on multi-valued mappings on metric spaces. Also Cho(2016), Jinakul et al. (2017) have worked on multi-valued mappings in b-metric spaces. Abdou (2016) introduced the notion CLRf -property and OWC-property. Djoudi and Khemis(2005) have introduced D -mappings in metric spaces. Gahler(1963) introduced the concept of 2-metric spaces. Abd El-Monsef et al. (2007) have worked on 2-metric spaces using multi-valued mappings and have defined $\delta$. Various authors have used the notion of $\mathrm{D}, \mathrm{H}, \delta$ to obtain their results in metric spaces. In the present role we have used these symbols with their usual meanings to obtain our results in 2-metric space.

\section{DEFINITIONS}

Definition 2.1 [9]: Let $\mathrm{X}$ be a non-empty set and $\mathrm{d}$ : $\mathrm{X} \times \mathrm{X}$ $x X \rightarrow[0, \infty)$ be a real valued function which satisfy the following conditions:

1. For every distinct points $\mathrm{x}, \mathrm{y}$ there is a point $\mathrm{z}$ in $\mathrm{X}$ such that $d(x, y, z) \neq 0$;

2. $d(x, y, z)=0$ if any two of three of $x, y, z$ is equal;

3. $d(x, y, z)=d(p(x, y, z))$ for all $x, y, z \in X$ and for all permutations $\mathrm{p}(\mathrm{x}, \mathrm{y}, \mathrm{z})$ of $\mathrm{x}, \mathrm{y}, \mathrm{z}$;

4. $d(x, y, z) \leq d(x, y, w)+d(x, w, z)+d(w, y, z)$ for all $x$, $\mathrm{y}, \mathrm{z}, \mathrm{w} \in \mathrm{X}$.

Then $\mathrm{d}$ is called a 2-metric and (X, d) is called a 2-metric space.

We write $X$ a 2-metric space unless otherwise stated. Here by $\mathrm{CB}(\mathrm{X})$ we mean the class of all nonempty closed and bounded subsets of $\mathrm{X}$, by $2^{\mathrm{X}}$ we mean the class of all nonempty compact subsets of $\mathrm{X}$ and by $\mathrm{B}(\mathrm{X})$ we mean the class of all bounded subsets of $\mathrm{X}$. 
For all $A, B, C$ in $B(X)$ we define the following definitions:

Definition 2.2 [2]. Let $\delta: X \times X \times X \rightarrow[0, \infty)$ be defined as $\delta(A, B, C)=\sup \{d(a, b, c): a \in A, b \in B, c \in C\}$.

If $\mathrm{A}=\{\mathrm{a}\}$ we write $\delta(\mathrm{A}, \mathrm{B}, \mathrm{C})=\delta(\mathrm{a}, \mathrm{B}, \mathrm{C})$.

If $A=\{a\}, B=\{b\}$ then we write $\delta(A, B, C)=\delta(a, b, C)$.

If $A=\{a\}, B=\{b\}, C=\{c\}$ then we write $\delta(A, B, C)$ $=\delta(a, b, c)=d(a, b, c)$.

From the definition of $\delta$ it follows that for $A, B, C, E \in B(X)$ :

1. $\delta(\mathrm{A}, \mathrm{B}, \mathrm{C}) \geq 0$;

2. $\delta(\mathrm{A}, \mathrm{B}, \mathrm{C})=0$ if at least two of $\mathrm{A}, \mathrm{B}, \mathrm{C}$ consist of equal single point;

3. $\delta(A, B, C)=\delta(p(A, B, C))$ where $p(A, B, C)$ is the permutation of $A, B, C$;

4. $\delta(\mathrm{A}, \mathrm{B}, \mathrm{C}) \leq \delta(\mathrm{A}, \mathrm{B}, \mathrm{E})+\delta(\mathrm{A}, \mathrm{E}, \mathrm{C})+\delta(\mathrm{E}, \mathrm{B}, \mathrm{C})$.

With this definition we say that $\delta$ is a multi-valued 2- metric on $\mathrm{B}(\mathrm{X})$.

Clearly, $\mathrm{d} \leq \delta$.

We now introduce the following definition.

Definition 2.3: For all $A, B, C \in C B(X)$, let us consider a function $\mathrm{D}: \mathrm{X} \times \mathrm{X} \times \mathrm{X} \rightarrow[0, \infty)$ defined by

$D(A, B, C)=\inf \{d(a, b, c): a \in A, b \in B, c \in C\}$.

If $A=\{a\}$, we write $D(A, B, C)=D(a, B, C)=\inf \{d(a, b, c)$ : $\mathrm{b} \in \mathrm{B}, \mathrm{c} \in \mathrm{C}\}$.

If $A=\{a\}, B=\{b\}$ then we write $D(A, B, C)=D(a, b, C)$ $=\inf \{d(a, b, c): c \in C\}$.

If $A=\{a\}, B=\{b\}, C=\{c\}$ then we write $D(A, B, C)=$ $D(a, b, c)=d(a, b, c)$.

From the definition of D it follows that for A, B, C, E $\in \mathrm{CB}(\mathrm{X})$ :

1. $\mathrm{D}(\mathrm{A}, \mathrm{B}, \mathrm{C}) \geq 0$;

2. $\mathrm{D}(\mathrm{A}, \mathrm{B}, \mathrm{C})=0$ if at least two of $\mathrm{A}, \mathrm{B}, \mathrm{C}$ consist of equal single point;

3. $D(A, B, C)=D(p(A, B, C))$ where $p(A, B, C)$ is the permutation of $A, B, C$;

4. $\quad \mathrm{D}(\mathrm{A}, \mathrm{B}, \mathrm{C}) \leq \mathrm{D}(\mathrm{A}, \mathrm{B}, \mathrm{E})+\mathrm{D}(\mathrm{A}, \mathrm{E}, \mathrm{C})+\mathrm{D}(\mathrm{E}, \mathrm{B}, \mathrm{C})$.

With this definition we say that $\mathrm{D}$ is another multivalued 2-metric on $\mathrm{CB}(\mathrm{X})$.

From the definition it is clear that $\mathrm{D} \leq \mathrm{d}$.
NOTE 2.1: In case of $A, B, C \in B(X)$ then the condition i) would be $D(A, B, C)=0$ if at least two of $A, B, C$ consist of equal single point or they have a non-empty intersection.

NOTE 2.2: From the definitions of $\delta, D$ and $d$, it is clear that $\mathrm{D} \leq \mathrm{d} \leq \delta$.

Again we are going to introduce another function $\mathrm{H}(\mathrm{A}, \mathrm{B}, \mathrm{C})$.

Definition 2.4: Let $A, B, C$ in $C B(X)$ and be pairwise disjoint. We define a function $\mathrm{H}(\mathrm{A}, \mathrm{B}, \mathrm{C})$ where $\mathrm{H}(\mathrm{A}, \mathrm{B}, \mathrm{C})$ : $\mathrm{X} \times \mathrm{X} \times \mathrm{X} \rightarrow[0, \infty)$ defined by

$H(A, B, C)=\max \left\{\sup _{a \in A} D(a, B, C),\left\{\sup _{b \in B} D(b, C, A)\right.\right.$, $\left.\sup _{c \in c} D(c, A, B)\right\}$.

Now,

1. Clearly $\mathrm{H}(\mathrm{A}, \mathrm{B}, \mathrm{C}) \geq 0$ and $\mathrm{H}(\mathrm{A}, \mathrm{B}, \mathrm{C})=0$ i.e., $\max \left\{\sup _{\mathbf{a} \in \mathbf{A}} D(a, B, C),\left\{\sup _{\mathbf{b} \in \mathbf{B}} D(b, C, A), \sup _{\mathbf{c} \in \mathbf{C}}\right.\right.$ $\mathrm{D}(\mathrm{c}, \mathrm{A}, \mathrm{B})\}=0$ implies, $\sup _{\mathrm{a} \in \mathbf{A}} \mathrm{D}(\mathrm{a}, \mathrm{B}, \mathrm{C})=0, \sup _{\mathbf{b} \in \mathbf{B}}$ $\mathrm{D}(\mathrm{b}, \mathrm{C}, \mathrm{A})=0, \sup _{\mathrm{c} \in \mathrm{C}} \mathrm{D}(\mathrm{c}, \mathrm{A}, \mathrm{B})=0$.

Again, $\sup _{a \in A} D(a, B, C)=0$ implies, $\sup _{a \in A}\{\inf \{d(a, b, c)$ : $b \in B, c \in C\}\}=0$ i.e., inf $d(a, b, c)=0$, for all $a \in A$ i.e., $d(a, b, c)=0$, for all $a \in A$ i.e., $b=c$ i.e., $B=C$. Similarly, we can show that $\sup _{b \in \mathbf{B}} D(b, C, A)=0$ implies, $A=C$, and $\sup _{c \in C} D(c, A, B)=0$ implies, $A=B$. Thus $\mathrm{H}(\mathrm{A}, \mathrm{B}, \mathrm{C})=0$ if two of $\mathrm{A}, \mathrm{B}, \mathrm{C}$ are equal.

2. From definition it is clear that $H(A, B, C)=H(p(A, B, C))$ where $p(A, B, C)$ is the permutation of $A, B, C$;

3. Since $d$ is a 2-metric on $X$ we have for all $a \in A, b \in B$, $c \in C, e \in E d(a, b, c) \leq d(a, b, e)+d(a, e, c)+d(e, b, c)$

Taking infimum on the both sides we have

$\inf d(a, b, c) \leq \inf d(a, b, e)+\inf d(a, e, c)+\inf d(e, b, c)$ for all $a \in A, b \in B, c \in C, e \in E$, which implies, $\sup _{a \in A}\{\inf \{d(a, b, c):$ $\mathrm{b} \in \mathrm{B}, \mathrm{c} \in \mathrm{C}\}\} \leq \sup _{\mathrm{a} \in \mathrm{A}}\{\inf \{\mathrm{d}(\mathrm{a}, \mathrm{b}, \mathrm{e}): \mathrm{b} \in \mathrm{B}, \mathrm{e} \in \mathrm{E}\}\}+\sup _{\mathrm{a} \in \mathrm{A}}$ $\{\inf \{d(a, e, c): e \in E, c \in C\}\}+\sup _{e \in E}\{\inf \{d(e, b, c): b \in B$, $c \in C\}\}$ implies, $\sup _{a \in A} D(a, B, C) \leq \sup _{a \in A} D(a, B, E)+\sup _{a \in A}$ $\mathrm{D}(\mathrm{a}, \mathrm{E}, \mathrm{C})+\sup _{\mathbf{e} \in \mathbf{E}} \mathrm{D}(\mathrm{e}, \mathrm{B}, \mathrm{C})$ Similarly, we can show that $\sup _{b \in \mathbf{B}} D(b, C, A) \leq \sup _{b \in \mathbf{B}} D(b, C, E)+\sup _{b \in \mathbf{B}} D(b, E, A)+$ $\sup _{\mathbf{e} \in \mathbf{E}} \mathrm{D}(\mathrm{e}, \mathrm{C}, \mathrm{A})$ and $\sup _{\mathbf{c} \in \mathbf{C}} \mathrm{D}(\mathrm{c}, \mathrm{A}, \mathrm{B}) \leq \sup _{\mathbf{c} \in \mathrm{C}} \mathrm{D}(\mathrm{c}, \mathrm{A}, \mathrm{E})+$ $\sup _{c \in C} D(c, E, B)+\sup _{e \in E} D(e, A, B)$.

Therefore, $\max \left\{\sup _{a \in A} D(A, B, C) ; \sup _{b \in \mathbf{B}} D(B, C, A)\right.$; $\left.\sup _{\mathbf{c} \in \mathbf{c}} \mathrm{D}(\mathrm{C}, \mathrm{A}, \mathrm{B})\right\} \leq \max \left\{\sup _{\mathbf{a} \in \mathbf{A}} \mathrm{D}(\mathrm{a}, \mathrm{B}, \mathrm{E}) ; \sup _{\mathbf{b} \in \mathrm{B}} \mathrm{D}(\mathrm{b}, \mathrm{E}, \mathrm{A})\right.$; $\left.\sup _{\mathbf{e} \in \mathbf{E}} \mathrm{D}(\mathrm{e}, \mathrm{A}, \mathrm{B})\right\}+\max \left\{\sup _{\mathbf{a} \in \mathbf{A}} \mathrm{D}(\mathrm{a}, \mathrm{E}, \mathrm{C}) ; \sup _{\mathbf{e} \in \mathrm{E}} \mathrm{D}(\mathrm{e}, \mathrm{C}, \mathrm{A})\right.$; $\left.\sup _{\mathbf{c} \in \mathbf{c}} D(c, A, E)\right\}+\max \left\{\sup _{\mathbf{e} \in E} D(e, B, C) ; \sup _{\mathbf{b} \in \mathbf{B}} D(b, C, E)\right.$; $\left.\sup _{c \in c} D(c, E, B)\right\}$ implies, H(A, B, C) $\leq H(A, B, E)+H(A, E, C)$ $+\mathrm{H}(\mathrm{E}, \mathrm{B}, \mathrm{C})$. 
Therefore $\mathrm{H}$ is a 2-metric called 2-Hausdorff metric and $(\mathrm{CB}(\mathrm{X}), \mathrm{H})$ is called 2-Hausdoff metric space. From the definition we have $\mathrm{D} \leq \mathrm{H}$.

Lemma 21: If $A, B, C \in C B(X)$, then $H \leq \delta$.

Proof. We know $D(A, B, C)=\inf \{d(a, b, c): a \in A, b \in B$, $\in \in C\}$ and $H(A, B, C)=\max \left\{\sup _{a \in A} D(a, B, C) ; \sup _{b \in B} D(b, C, A)\right.$; $\left.\sup _{\mathbf{c} \in \mathbf{C}} \mathrm{D}(\mathrm{c}, \mathrm{A}, \mathrm{B})\right\}$.

CASE I: If $A=\{a\}, B=\{b\}, C=\{c\}$ then $D(a, b, c)=$ $\mathrm{D}(\mathrm{b}, \mathrm{c}, \mathrm{a})=\mathrm{D}(\mathrm{c}, \mathrm{a}, \mathrm{b})=\mathrm{d}(\mathrm{a}, \mathrm{b}, \mathrm{c})=\delta(\mathrm{A}, \mathrm{B}, \mathrm{C})$ and so $\mathrm{H}(\mathrm{A}, \mathrm{B}, \mathrm{C})$ $=\delta(A, B, C)$.

CASE II: If $A=\{a\}, B=\{b\}$ then $D(a, b, C)=\inf \{d(a, b, c)$ : $c \in C\} \leq \inf d(a, b, c) \leq \sup _{c \in C} d(a, b, c)=\delta(A, B, C)$ implies, $\sup _{c \in C} D(A, B, C)=\sup _{c \in C} D(a, b, C) \leq \delta(A, B, C)$ So $H(A, B, C)$ $\leq \delta(\mathrm{A}, \mathrm{B}, \mathrm{C})$.

CASE III: If $A=\{a\}$, then $D(a, B, C)=\inf \{d(a, b, c): b \in B$, $c \in C\}$ and suppose $H(A, B, C)=\sup _{a \in A} D(a, B, C)$.

Since, $\inf \{d(a, b, c): b \in B, c \in C\} \leq\{d(a, b, c): b \in B, c \in C\}$ $\leq \sup \{d(a, b, c): b \in B, c \in C\}$ implies, $D(a, B, C) \leq \delta(a, B, C)$ implies, $\sup _{a \in A} D(a, B, C) \leq \delta(a, B, C)$ i.e., $H(A, B, C) \leq \delta(A, B, C)$.

CASE IV: If $A, B, C$ are not single point sets, then $\inf \left\{d_{a \in A}\right.$ $(a, B, C): b \in B, c \in C\} \leq \inf \{d(a, B, C): b \in B, c \in C\}$ i.e., $\mathrm{D}(\mathrm{A}, \mathrm{B}, \mathrm{C})=\mathrm{D}_{\mathrm{a} \in \mathrm{A}}(\mathrm{a}, \mathrm{B}, \mathrm{C}) \leq \delta(\mathrm{A}, \mathrm{B}, \mathrm{C})$ implies, $\left\{\sup _{\mathrm{a} \in \mathrm{A}} \mathrm{D}(, \mathrm{B}, \mathrm{C})\right.$ $\leq \delta(A, B, C)$. Similarly $\sup _{b \in B} D(b, C, A) \leq \delta(B, C, A)$ and $\sup _{c \in C}$ $\mathrm{D}(\mathrm{c}, \mathrm{A}, \mathrm{B}) \leq \delta(\mathrm{C}, \mathrm{A}, \mathrm{B})$.

Thus $\mathrm{H}(\mathrm{A}, \mathrm{B}, \mathrm{C}) \leq \delta(\mathrm{A}, \mathrm{B}, \mathrm{C})$.

Nadler(1969) has defined multi-valued contraction mapping on metric space. We are to define it in 2-metric spaces.

Definition 2.5. A sequence $\left\{x_{n}\right\}$ is said to converge to $x$ in $(X, D)$ if $\lim _{n \rightarrow \infty} x_{n}=x$ i.e., $\lim _{n \rightarrow \infty} D\left(x_{n}, x, a\right)=0$.

Definition 2.6. Let $(X, d)$ be a complete 2-metric space and $\mathrm{F}: \mathrm{X} \rightarrow \mathrm{CB}(\mathrm{X})$. Then $\mathrm{F}$ is said to be a multi-valued contraction mapping on 2-metric space if there exists a constant $\alpha$ where $0<\alpha<1$ such that $\mathrm{H}(\mathrm{F}(\mathrm{x}), \mathrm{F}(\mathrm{y}), \mathrm{a}) \leq \alpha \mathrm{d}(\mathrm{x}, \mathrm{y}, \mathrm{a})$.

Definition 2.7. [Jungck(1986)]. Let (X, d) be a 2-metric space. A single valued mapping $\mathrm{f}: \mathrm{X} \rightarrow \mathrm{X}$ and a multi-valued mapping $\mathrm{S}: \mathrm{X} \rightarrow \mathrm{CB}(\mathrm{X})$ are said to be weakly compatible if they commute at their coincidence points, i.e., if $\mathrm{fSx}=\mathrm{Sfx}$ whenever $\mathrm{fx} \in \mathrm{Sx}$.

Definition 2.8. [Sintunavarat and Kumar (2011)]. Let $(\mathrm{X}, \mathrm{d})$ be a 2-metric space. Two mappings $\mathrm{f}, \mathrm{g}: \mathrm{X} \rightarrow \mathrm{X}$ are said to satisfy the common limit in the range of $\mathrm{f}$ with respect to $\mathrm{g}$ (shortly, the $(\mathrm{CLR})_{\mathrm{f}}$ - property with respect to $\mathrm{g}$ ) if there exists a sequence $\left\{x_{n}\right\}$ in $X$ such that

$$
\lim _{n \rightarrow \infty} \mathrm{fx}_{\mathrm{n}}=\lim _{n \rightarrow \infty} \mathrm{gx}_{\mathrm{n}}=\mathrm{fu} \text {, for some } \mathrm{u} \text { in } \mathrm{X} \text {. }
$$

We shall exhibit in the following example the existence of two mappings which satisfy $\mathrm{CLR}_{\mathrm{f}}$ - property.

Example 2.1: Let $\mathrm{X}=\mathrm{R}+$ and $\mathrm{d}: \mathrm{X} \times \mathrm{X} \times \mathrm{X} \rightarrow \mathrm{R}_{+}$defined by $d(x, y, a)=\min \{|x-y|,|y-z|,|z-x|\}$. Then clearly $(X, d)$ is 2-metric space. Let $\mathrm{f}$; $\mathrm{g}: \mathrm{X} \rightarrow \mathrm{X}$ be given by $\mathrm{fx}=\frac{\mathrm{x}}{\mathrm{k}}$ and $\mathrm{gx}$ $=\frac{\mathrm{x}}{\mathrm{m}}$ where $\mathrm{k} \neq \mathrm{m} \in \mathrm{N}$ for all $\mathrm{x} \in \mathrm{X}$. Consider the sequence $\left\{x_{n}\right\}=\left\{\frac{1}{n}\right\}$ for each $n \in$ N. Clearly $\lim _{n \rightarrow \infty} \mathrm{fx}_{\mathrm{n}}=\lim _{n \rightarrow \infty} \mathrm{gx}_{\mathrm{n}}=\mathrm{f}(0)$. Therefore $\mathrm{f}$, g satisfies the (CLR) $)_{\mathrm{f}}$ - property with respect to $\mathrm{g}$.

Definition 2.9. [1]: Let $(X, d)$ be a 2-metric space. Two mappings $\mathrm{f}: \mathrm{X} \rightarrow \mathrm{X}$ and $\mathrm{S}: \mathrm{X} \rightarrow \mathrm{CB}(\mathrm{X})$ are said to be occasionally weakly compatible(shortly, (OWC)-property) if fS $\subset \subset$ Sfx for some $\mathrm{x} \in \mathrm{X}$ with $\mathrm{fx} \in \mathrm{Sx}$.

Definition 2.10. [8]: Let (X, d) be a 2-metric space. Then two mappings $\mathrm{f}: \mathrm{X} \rightarrow \mathrm{X}$ and $\mathrm{S}: \mathrm{X} \rightarrow \mathrm{CB}(\mathrm{X})$ are said to satisfy D-mapping if there exists a sequence $\left\{x_{n}\right\}$ in $X$ such that $\lim _{n \rightarrow \infty} f x_{n}=z$ and $\lim _{n \rightarrow \infty} S x_{n}=\{z\}$.

Example 2.2: Let $\mathrm{X}=[0, \infty)$ and $\mathrm{F}: \mathrm{X} \rightarrow \mathrm{B}(\mathrm{X}), \mathrm{f}: \mathrm{X} \rightarrow \mathrm{X}$ defined by $F x=\left[0, x^{2}\right]$ and $f x=\frac{x}{k} ; k \in N$ for all $x \in X$. Consider the sequence $\left\{x_{n}\right\}=\left\{\frac{1}{n}\right\}$ for all $n \in N$. Then clearly, $\lim _{n \rightarrow \infty} \mathrm{fx}_{\mathrm{n}}$ $=0$ and $\lim _{n \rightarrow \infty} f x_{n}=f\{0\}$.

Definition 2.11. Let $(X, d)$ be a 2-metric space. A mapping $\mathrm{T}: \mathrm{X} \rightarrow \mathrm{X}$ is said to be an $\mathrm{F}$ - contraction if there exists $\tau \in \mathbf{R}_{+}$and a function $F \in \mathbf{F}$ such that for all $x, y \in X, d(T x, T y, a)>0$ implies, $\tau+F(d(T x, T y, a)) \leq$ $F(d(x, y, a))$.

Definition 2.12. [Ahmad et al., 2015] A function F: $\mathbf{R}_{+} \rightarrow \mathbf{R}_{+}$be such that for $\mathrm{h}>1, \mathrm{~F}(\mathrm{hH}(\mathrm{Tx}, \mathrm{Ty}, \mathrm{a})) \leq \mathrm{F}(\mathrm{H}(\mathrm{Tx}$, $\mathrm{Ty}, \mathrm{a})$ ). Then $\mathrm{F}$ is called a function continuous from right.

\section{MAIN RESULTS}

Nadler(1969) proved the following theorem:

Theorem: Let $(X, d)$ be a complete metric space. If $\mathrm{F}: \mathrm{X} \rightarrow \mathrm{CB}(\mathrm{X})$ is a multi-valued contraction mapping, then $\mathrm{F}$ has a fixed point. 
We have generalized it to 2-metric space as below:

Theorem 3.1. If $X$ is a complete 2-metric space and $\mathrm{F}: \mathrm{X} \rightarrow \mathrm{CB}(\mathrm{X})$ is a multi-valued contraction mapping, then $\mathrm{F}$ has a fixed point in $X$.

Proof. Let $\mathrm{x}_{0}$ be arbitrary. Since $\mathrm{F}$ is a multi-valued contraction mapping, there is a positive constant $\varepsilon<1$ such that $H(F(x), F(y), a) \leq \varepsilon d(x, y, a)$.

Now choose a point $x_{1} \in F\left(x_{0}\right)$. Since $F\left(x_{0}\right)$ and $F\left(x_{1}\right)$ are in $\mathrm{CB}(\mathrm{X})$, for $\mathrm{x}_{1} \in \mathrm{F}\left(\mathrm{x}_{0}\right)$ there is a point $\mathrm{x}_{2} \in \mathrm{F}\left(\mathrm{x}_{1}\right)$ such that $d\left(x_{1}, x_{2}, a\right) \leq H\left(F\left(x_{0}\right), F\left(x_{1}\right), a\right)+\varepsilon$

Again since $\mathrm{F}\left(\mathrm{x}_{1}\right), \mathrm{F}\left(\mathrm{x}_{2}\right) \in \mathrm{CB}(\mathrm{X})$ and $\mathrm{x}_{2} \in \mathrm{F}\left(\mathrm{x}_{1}\right)$ there is a point $\mathrm{x}_{3} \in \mathrm{F}\left(\mathrm{x}_{2}\right)$ such that $\mathrm{d}\left(\mathrm{x}_{2}, \mathrm{x}_{3}, \mathrm{a}\right) \leq \mathrm{H}\left(\mathrm{f}\left(\mathrm{x}_{1}\right), \mathrm{F}\left(\mathrm{x}_{2}\right), \mathrm{a}\right)+\varepsilon^{2}$

Considering that way we can get a sequence $\left\{\mathbf{x}_{n}\right\}$ in $X$ such that $d\left(x_{n}, x_{n+1}, a\right) \leq H\left(F\left(x_{n-1}\right), F\left(x_{n}\right), a\right)+\varepsilon^{n}$

Now

$$
\begin{aligned}
d\left(x_{n}, x_{n+1}, a\right) & \leq H\left(F\left(x_{n-1}\right), F\left(x_{n}\right), a\right)+\varepsilon^{n} \\
& \leq \varepsilon d\left(x_{n-1}, x_{n}, a\right)+\varepsilon^{n} \\
& \leq \varepsilon\left(H\left(F\left(x_{n-2}\right), F\left(x_{n-1}\right), a\right)+\varepsilon^{n-1}\right)+\varepsilon^{n} \\
& =\varepsilon\left(H\left(F\left(x_{n-2}\right), F\left(x_{n-1}\right), a\right)+2 \varepsilon^{n}\right. \\
& \leq \varepsilon^{2} d\left(x_{n-2}, x_{n-1}, a\right)+2 \varepsilon^{n} \\
& \cdot \\
& \cdot \\
& \cdot \\
& \leq \varepsilon^{n} d\left(x_{0}, x_{1}, a\right)+n \varepsilon^{n} .
\end{aligned}
$$

Taking limit as $\mathrm{n} \rightarrow \infty$ on the both side of the above inequality we get $\lim _{n \rightarrow \infty} d\left(x_{n}, x_{n}+1, a\right)=0$ [as $\left.\varepsilon<1\right]$

$$
\text { So } \lim _{n \rightarrow \infty} d\left(x_{n}, x_{m}, x_{n-1}\right)=0 \text {. }
$$

Thus for $\mathrm{n} \geq \mathrm{m} \in \mathrm{N} ; \lim _{n, m \rightarrow \infty} \mathrm{d}\left(\mathrm{x}_{\mathrm{n}}, \mathrm{x}_{\mathrm{m}}, \mathrm{a}\right) \leq \lim _{n, m \rightarrow \infty}\left(\mathrm{d}\left(\mathrm{x}_{\mathrm{n}}, \mathrm{x}_{\mathrm{m}}, \mathrm{x}_{\mathrm{n}-1}\right)\right.$ $\left.+d\left(x_{n}, x_{n-1}, a\right)+d\left(x_{n-1}, x_{m}, a\right)\right)=\lim _{n, m \rightarrow \infty} d\left(x_{n-1}, x_{m}, a\right) \leq \ldots \leq \lim _{n, m \rightarrow \infty}$ $d\left(x_{m}, x_{m}, a\right)=0$.

Therefore $\left\{x_{n}\right\}$ is a Cauchy sequence in $X$. Since $X$ is complete, there exists an $\mathrm{x} \in \mathrm{X}$ such that $\mathrm{x}_{\mathrm{n}} \rightarrow \mathrm{x}$ as $\mathrm{n} \rightarrow \infty$. So $\left\{F\left(x_{n}\right)\right\}$ converges to $F(x)$ and hence $x \in F(x)$. Therefore $x$ is a fixed point of $\mathrm{F}$ in $\mathrm{X}$.

Note 3.1: If $A, B \in C B(X)$ and $a \in A$ then from (3.1) of the above theorem we get $\mathrm{b} \in \mathrm{B}$ such that $\mathrm{d}(\mathrm{a}, \mathrm{b}, \mathrm{x}) \leq \mathrm{H}(\mathrm{A}, \mathrm{B}, \mathrm{x})+$ $\alpha, 0<\alpha<1$.
Theorem 3.2. Let $(X, d)$ be a 2-metric space. $f, g: X \rightarrow X$ and $\mathrm{S}, \mathrm{T}: \mathrm{X} \rightarrow \mathrm{CB}(\mathrm{X})$ satisfying the relation,

$\operatorname{Hp}(\mathrm{Sx}, \mathrm{Ty}, \mathrm{a})$

$\leq \alpha_{1} \mathrm{~d}^{\mathrm{p}}(\mathrm{fx}, \mathrm{gy}, \mathrm{a})+\alpha_{2} \delta^{\mathrm{p}}(\mathrm{fx}, \mathrm{Ty}, \mathrm{a})+\alpha_{3} \delta^{\mathrm{p}}(\mathrm{gy}, \mathrm{Sx}, \mathrm{a})+\alpha_{4} \delta^{\mathrm{p}}(\mathrm{gy}, \mathrm{Ty}$, a) $+\alpha_{5} \delta^{p}(\mathrm{fx}, \mathrm{Sx}, \mathrm{a})$, where $\mathrm{p} \geq 1, \alpha_{\mathrm{i}} \geq 0, \mathrm{i}=1,2,3,4,5$ and $\sum_{i=1}^{5} \alpha_{i}<\frac{1}{n^{p}}, \mathrm{n} \in \mathrm{N}$. The pairs (S, f) and (T, g) satisfy OWCproperty; $f(X)$ and $g(X)$ are closed. Then $f, g, S$ and $T$ have unique common fixed point in $\mathrm{X}$.

Proof. Since the pair (S, f) and (T, g) satisfy OWCproperty, there exist $u, v \in X$ such that fu $\in \mathrm{Su}, \mathrm{fSu} \subset \mathrm{Sfu}$, gv $\in$ Tv, gTv $\subset$ Tgv which implies, ffu $\in$ Sfu and ggv $\in$ Tgv.

Now we show that $f u=g v$. Let us suppose that $f u \neq g v$. Then, $D^{p}(f u, g v, a) \leq H^{p}(S u, T v, a) \leq \alpha_{1} d^{p}(f u, g v, a)+\alpha_{2} \delta^{p}(f u, T v, a)+\alpha_{3}$ $\delta^{\mathrm{p}}(\mathrm{gv}, \mathrm{Su}, \mathrm{a})+\alpha_{4} \delta^{\mathrm{p}}(\mathrm{gv}, \mathrm{Tv}, \mathrm{a})+\alpha_{5} \delta^{\mathrm{p}}(\mathrm{fu}, \mathrm{Su}, \mathrm{a}) \leq \alpha_{1} \mathrm{dp}(\mathrm{fu}, \mathrm{gv}, \mathrm{a})$ $+\alpha_{2} \delta^{\mathrm{p}}(\mathrm{Su}, \mathrm{Tv}, \mathrm{a})+\alpha_{3} \delta^{\mathrm{p}}(\mathrm{Tv}, \mathrm{Su}, \mathrm{a})+\alpha_{4} \delta^{\mathrm{p}}(\mathrm{Tv}, \mathrm{Tv}, \mathrm{a})+\alpha_{5} \delta^{\mathrm{p}}(\mathrm{Su}, \mathrm{Su}, \mathrm{a})$ $\leq\left(\alpha_{1}+\alpha_{2}+\alpha_{3}\right) \delta^{\mathrm{p}}(\mathrm{Su}, \mathrm{Tv}, \mathrm{a})$

Since $\mathrm{D} \leq \delta ; \mathrm{D}, \delta \in \mathrm{R}_{+}=[0, \infty)$ there exists $\mathrm{n} \in \mathrm{N}$ such that $\delta \leq \mathrm{nD}$ [by Archimedean Property], i.e., $\delta^{\mathrm{p}}(\mathrm{Su}, \mathrm{Tv}, \mathrm{a}) \leq$ $\mathrm{n}^{\mathrm{p}} \mathrm{D}^{\mathrm{p}}(\mathrm{fu}, \mathrm{gv}, \mathrm{a})$ i.e., $\left(\alpha_{1}+\alpha_{2}+\alpha_{3}\right) \delta \mathrm{p}(\mathrm{Su}, \mathrm{Tv}, \mathrm{a}) \leq\left(\alpha_{1}+\alpha_{2}+\alpha_{3}\right)$ $n^{p} D^{p}(f u, g v, a)$.

From (2.3) we get $D^{p}(f u, g v, a) \leq\left(\alpha_{1}+\alpha_{2}+\alpha_{3}\right) n^{p} D^{p}(f u, g v, a)$ implies, $\mathrm{D}^{\mathrm{p}}(\mathrm{fu}, \mathrm{gv}, \mathrm{a})=0\left[\right.$ since $\left.\left(\alpha_{1}+\alpha_{2}+\alpha_{3}\right) \mathrm{n}^{\mathrm{p}}<1\right]$ i.e., $\mathrm{fu}=\mathrm{gv}$.

Now we are to show that fu is fixed point of $f$. So, $D^{p}(f f u, f u, a)=D^{p}(f f u, g v, a) \leq H^{p}(S f u, T v, a) \leq \alpha_{1} d(f f u, g v, a)$ $+\alpha_{2} \delta$ (ffu, Tv, a) $+\alpha_{3} \delta$ (gv, Sfu, a) $+\alpha_{4} \delta^{\mathrm{p}}(\mathrm{gv}, \mathrm{Tv}, \mathrm{a})+\alpha_{5} \delta^{\mathrm{p}}(\mathrm{ffu}$, $\mathrm{Sfu}, \mathrm{a})=\alpha_{1} \mathrm{~d}^{\mathrm{p}}(\mathrm{ffu}, \mathrm{gv}, \mathrm{a})+\alpha_{2} \delta^{\mathrm{p}}(\mathrm{ffu}, \mathrm{Tv}, \mathrm{a})+\alpha_{3} \delta^{\mathrm{p}}(\mathrm{Sfu}, \mathrm{gv}, \mathrm{a})=$ $\alpha_{1} d^{p}(f f u, g v, a)+\alpha_{2} \delta^{p}(f f u, T v, a)+\alpha_{3} \delta^{p}(S f u, f u, a)$

$$
=\left(\alpha_{1}+\alpha_{2}+\alpha_{3}\right) \delta^{p}(S u, \text { Tv. } a)
$$

Again we know that $\mathrm{D}(\mathrm{ffu}, \mathrm{gv}, \mathrm{a}) \leq \delta(\mathrm{Sfu}, \mathrm{Tv}, \mathrm{a})$ and $\mathrm{D}(\mathrm{ffu}, \mathrm{gv}, \mathrm{a}), \delta(\mathrm{Sfu}, \mathrm{Tv}, \mathrm{a}) \in \mathrm{R}+$ by Archimedean Property, there exists a $n_{0} \in N$ such that $\delta(S f u, T v, a) \leq n_{0} D(f f u, g v, a)$ implies, $\delta^{\mathrm{p}}(\mathrm{Sfu}, \mathrm{Tv}, \mathrm{a}) \leq \mathrm{n}_{0}{ }^{\mathrm{p}} \mathrm{D}^{\mathrm{p}}(\mathrm{ffu}, \mathrm{gv}, \mathrm{a})$.

From (2.4) $D^{p}(f f u, f u, a) \leq\left(\alpha_{2}+\alpha_{2}+\alpha_{3}\right) n_{0}{ }^{p} D^{p}(f f u, g v, a)=$ $\left(\alpha_{1}+\alpha_{2}+\alpha_{3}\right) n_{0}{ }^{p} D^{p}(f f u, f u, a)$ [since fu $=$ gv] implies, $D^{p}(f f u, f u, a)$ $\leq 0$ i.e., $D^{p}(f f u ; f u ; a)=0$ i.e., $f f u=f u$ i.e., fu is a fixed point of $f$.

Now we are to show that fu is also a fixed point of $g$. Since $D(f u, T g v, a) \leq d(f u, g g v, a)$, by Archimedean property there exist an $n^{*} \in N$ such that $d(f u, g f u, a) \leq n * D(f u, T f u, a)$ i.e., $d^{p}(f u, g f u, a) \leq n^{* p} D^{p}(f u, T f u, a)$. 
So, $D^{p}(f u, g f u, a)=d^{p}(f u, g g v, a) \leq n^{* p} D^{p}(f u, T g v, a) \leq$ $n^{* p} H^{p}(S f u, T g v, a) \leq n^{* p}\left[\alpha_{1} d^{p}(f f u, g g v, a)+\alpha_{2} \delta^{p}(f f u, T g v, a)\right.$ $\left.+\alpha_{3} \delta^{\mathrm{p}}(\mathrm{ggv}, \mathrm{Sfu}, \mathrm{a})+\alpha_{4} \delta^{\mathrm{p}}(\mathrm{ggv}, \mathrm{Tgv}, \mathrm{a})+\alpha_{5} \delta^{\mathrm{p}}(\mathrm{ffu}, \mathrm{Sfu}, \mathrm{a})\right]$ $\leq\left(\alpha_{1}+\alpha_{2}+\alpha_{3}\right) n^{* p} \delta^{p}(\mathrm{Sfu}, \mathrm{Tgv}, \mathrm{a}) \leq\left(\alpha_{1}+\alpha_{2}+\alpha_{3}\right) \mathrm{n}^{* \mathrm{p}} \mathrm{n}_{00}$ ${ }^{\mathrm{p}} \mathrm{D}^{\mathrm{p}}(\mathrm{fu}, \mathrm{ggv}$, a) [similarly using Archimedean Property for $\left.\mathrm{n}_{00} \in \mathrm{N}\right]=\left(\alpha_{1}+\alpha_{2}+\alpha_{3}\right) \mathrm{k}^{\mathrm{p}}\left[\right.$ putting $\left.\mathrm{n}^{* \mathrm{p}} \mathrm{n}_{00}{ }^{\mathrm{p}} \mathrm{D}^{\mathrm{p}}=\mathrm{k}^{\mathrm{p}}\right]$ i.e., (1- $\left.\left(\alpha_{1}+\alpha_{2}+\alpha_{3}\right) k^{p}\right) D^{p}(f u, g f u, a) \leq 0$ [since fu = gv] implies, $D^{p}(f u, g f u, a)=0$ i.e., fu $=g f u$.

Therefore, $\mathrm{fu}=\mathrm{ffu}=\mathrm{gfu}=\mathrm{ggv} \in \mathrm{Tgv}=\mathrm{Tfu}$ and $\mathrm{fu}=\mathrm{ffu} \in \mathrm{Sfu}$. Thus $\mathrm{fu}=\mathrm{z}$ (say) is a common fixed point of $\mathrm{f}, \mathrm{g}, \mathrm{S}$ and $\mathrm{T}$.

Now $\mathrm{H}^{\mathrm{p}}(\mathrm{Sz}, \mathrm{Tz}, \mathrm{a})=\mathrm{H}^{\mathrm{p}}(\mathrm{Sfu}, \mathrm{Tfu}, \mathrm{a})=\mathrm{H}^{\mathrm{p}}(\mathrm{Sfu}, \mathrm{Tgv}, \mathrm{a})$ $\leq \alpha_{1} \mathrm{~d}^{\mathrm{p}}(\mathrm{ffu}, \mathrm{ggv}, \mathrm{a})+\alpha_{2} \delta^{\mathrm{p}}(\mathrm{ffu}, \mathrm{Tgv}, \mathrm{a})+\alpha_{3} \delta^{\mathrm{p}}(\mathrm{ggv}, \mathrm{Sfu}, \mathrm{a})+$ $\alpha_{4} \delta^{p}(g g v, T g v, a)+\alpha_{5} \delta^{p}(f f u, S f u, a)=\alpha_{1} \cdot 0+\alpha_{2} \cdot 0+\alpha_{3} \cdot 0+\alpha_{4} \cdot 0+$ $\alpha_{5} .0=0$,i.e., $\mathrm{Sz}=\mathrm{Tz}$.

Let $\mathrm{w}$ be another common fixed point of $\mathrm{f}, \mathrm{g}, \mathrm{S}$ and $\mathrm{T}$. Then we have $\mathrm{D}^{\mathrm{p}}(\mathrm{z}, \mathrm{w}, \mathrm{a}) \leq \mathrm{H}^{\mathrm{p}}(\mathrm{Sz}, \mathrm{Tw}, \mathrm{a}) \leq \alpha_{1} \mathrm{~d}^{\mathrm{p}}(\mathrm{fz}, \mathrm{gw}, \mathrm{a})+$ $\alpha_{2} \delta^{\mathrm{p}}(\mathrm{fz}, \mathrm{Tw}, \mathrm{a})+\alpha_{3} \delta^{\mathrm{p}}(\mathrm{gw}, \mathrm{Sz}, \mathrm{a})+\alpha_{4} \delta^{\mathrm{p}}(\mathrm{gw}, \mathrm{Tw}, \mathrm{a})+\alpha_{5} \delta^{\mathrm{p}}(\mathrm{fz}, \mathrm{Sz}, \mathrm{a})$ $=\alpha_{1} \mathrm{~d}^{\mathrm{p}}(\mathrm{z}, \mathrm{w}, \mathrm{a})+\alpha_{2} \delta^{\mathrm{p}}(\mathrm{z}, \mathrm{Tw}, \mathrm{a})+\alpha_{3} \delta^{\mathrm{p}}(\mathrm{w}, \mathrm{Sz}, \mathrm{a}) \leq\left(\alpha_{1}+\alpha_{2}+\alpha_{3}\right)$ $\delta^{\mathrm{p}}(\mathrm{Sz}, \mathrm{Tw}, \mathrm{a}) \leq\left(\alpha_{1}+\alpha_{2}+\alpha_{3}\right) \mathrm{n}_{000}{ }^{\mathrm{p}} \mathrm{D}^{\mathrm{p}}(\mathrm{z}, \mathrm{w}, \mathrm{a})$ [using Archimedean Property similarly for $\left.\mathrm{n}_{000} \in \mathbf{N}\right]$ implies, $\mathrm{D}^{\mathrm{p}}(\mathrm{z}, \mathrm{w}, \mathrm{a})=0$ i.e., $\mathrm{z}=\mathrm{w}$. Thus $\mathrm{Sz}=\mathrm{Tz}=\{\mathrm{z}\}$. Hence the theorem.

Corollary 3.1. Let $(X, d)$ be a 2-metric space. $F, g: X \rightarrow X$ and $\mathrm{S}, \mathrm{T}: \mathrm{X} \rightarrow \mathrm{CB}(\mathrm{X})$ satisfying the relation, $\mathrm{H}^{\mathrm{p}}(\mathrm{Sx}, \mathrm{Ty}, \mathrm{a}) \leq$ $\alpha_{1} \mathrm{~d}^{\mathrm{p}}(\mathrm{fx}, \mathrm{gy}, \mathrm{a})+\alpha_{2} \mathrm{D}^{\mathrm{p}}(\mathrm{fx}, \mathrm{Ty}, \mathrm{a})+\alpha_{3} \mathrm{D}^{\mathrm{p}}(\mathrm{gy}, \mathrm{Sx}, \mathrm{a})+\alpha_{4} \mathrm{D}^{\mathrm{p}}(\mathrm{gy}, \mathrm{Ty}$, a) $+\alpha_{5} \mathrm{D}^{\mathrm{p}}(\mathrm{fx}, \mathrm{Sx}, \mathrm{a}), \mathrm{p} \geq 1, \alpha_{\mathrm{i}} \geq 0, \mathrm{i}=1,2,3,4,5$ and $\sum_{i=1}^{5} \alpha \alpha_{\mathrm{i}}<1$. The pairs $(\mathrm{S}, \mathrm{f})$ and $(\mathrm{T}, \mathrm{g})$ satisfy OWC-property. $\mathrm{f}(\mathrm{X})$ and $\mathrm{g}(\mathrm{X})$ are closed. Then $\mathrm{f}, \mathrm{g}, \mathrm{S}$ and $\mathrm{T}$ have unique common fixed point in $\mathrm{X}$.

Proof. Since, $H^{p}(S x, T y, a) \leq \alpha_{1} d^{p}(f x, g y, a)+\alpha_{2} D^{p}(f x, T y$ a $)$ $+\alpha_{3} \mathrm{D}^{\mathrm{p}}(\mathrm{gy}, \mathrm{Sx}, \mathrm{a})+\alpha_{4} \mathrm{D}^{\mathrm{p}}(\mathrm{gy}, \mathrm{Ty}, \mathrm{a})+\alpha_{5} \mathrm{D}^{\mathrm{p}}(\mathrm{fx}, \mathrm{Sx}, \mathrm{a}) \leq \alpha_{1} \mathrm{~d}^{\mathrm{p}}(\mathrm{f} \mathrm{x}, \mathrm{gy}$, a) $+\alpha_{2} \delta^{p}(f x, T y, a)+\alpha_{3} \delta^{p}(g y, S x, a)+\alpha_{4} \delta^{p}(g y, T y, a)+\alpha_{5} \delta^{p}(f x, S x$, a), the result follows from Theorem 3.2.

Corollary 3.2. Let $(X, d)$ be a 2-metric space. $f, g: X \rightarrow X$ and $\mathrm{S}, \mathrm{T}: \mathrm{X} \rightarrow \mathrm{CB}(\mathrm{X})$ satisfying the relation, $\mathrm{H}^{\mathrm{p}}(\mathrm{Sx}, \mathrm{Ty}, \mathrm{a}) \leq$ $\alpha_{1} \mathrm{~d}^{\mathrm{p}}(\mathrm{x}, \mathrm{y}, \mathrm{a})+\alpha_{2} \mathrm{D}^{\mathrm{p}}(\mathrm{x}, \mathrm{Ty}, \mathrm{a})+\alpha_{3} \mathrm{D}^{\mathrm{p}}(\mathrm{y}, \mathrm{Sx}, \mathrm{a})+\alpha_{4} \mathrm{D}^{\mathrm{p}}(\mathrm{y}, \mathrm{Ty}, \mathrm{a})+$ $\alpha_{5} \mathrm{D}^{\mathrm{p}}(\mathrm{x}, \mathrm{Sx}, \mathrm{a})$; where $\mathrm{p} \geq 1, \alpha_{\mathrm{i}} \geq 0, \mathrm{i}=1,2,3,4,5$ and $\sum_{\mathrm{i}=1}^{5} \alpha \alpha_{\mathrm{i}}<1$. The pairs $(\mathrm{S}, \mathrm{f})$ and $(\mathrm{T}, \mathrm{g})$ satisfy OWC-property. $\mathrm{f}(\mathrm{X})$ and $\mathrm{g}(\mathrm{X})$ are closed. Then $\mathrm{f}, \mathrm{g}, \mathrm{S}$ and $\mathrm{T}$ have a unique common fixed point in X.

Proof. Since $D^{p}(S x, T y, a) \leq D^{p}(x, T y, a)$ and $D^{p}(S x, T y, a)$ $\leq \mathrm{D}^{\mathrm{p}}(\mathrm{S} x, \mathrm{y}, \mathrm{a})$, the result follows from Corollary 3.1.

Corollary 3.3. Let $(X, d)$ be a 2-metric space. F, $g: X \rightarrow X$ and $\mathrm{S}, \mathrm{T}: \mathrm{X} \rightarrow \mathrm{CB}(\mathrm{X})$ satisfying the relation, $\mathrm{D}^{\mathrm{p}}(\mathrm{Sx}, \mathrm{Ty}, \mathrm{a}) \leq$ $\alpha_{1} \mathrm{~d}^{\mathrm{p}}(\mathrm{fx}, \mathrm{gy}, \mathrm{a})+\alpha_{2} \mathrm{D}^{\mathrm{p}}(\mathrm{fx}, \mathrm{Ty}, \mathrm{a})+\alpha_{3} \mathrm{D}^{\mathrm{p}}(\mathrm{gy}, \mathrm{Sx}, \mathrm{a})+\alpha_{4} \mathrm{D}^{\mathrm{p}}(\mathrm{gy}, \mathrm{Ty}, \mathrm{a})$ $+\alpha_{5} \mathrm{D}^{\mathrm{p}}(\mathrm{fx}, \mathrm{Sx}, \mathrm{a}) ; \mathrm{p} \geq 1, \alpha_{\mathrm{i}} \geq 0, \mathrm{i}=1,2,3,4,5$ and $\sum_{i=1}^{5} \alpha \alpha_{\mathrm{i}}<1$. The pairs (S, f) and (T, g) satisfy OWC-property. $\mathrm{f}(\mathrm{X})$ and $\mathrm{g}(\mathrm{X})$ are closed. Then $\mathrm{f}, \mathrm{g}, \mathrm{S}$ and $\mathrm{T}$ have a unique common fixed point in X.

Proof. As we know that $\mathrm{D} \leq \mathrm{H}$ then we get, $\mathrm{D}^{\mathrm{p}}(\mathrm{Sx}, \mathrm{Ty}, \mathrm{a})$ $\leq \mathrm{H}^{\mathrm{p}}(\mathrm{Sx}, \mathrm{TY}, \mathrm{a}) \leq \alpha_{1} \mathrm{~d}^{\mathrm{p}}(\mathrm{fx}, \mathrm{gy}, \mathrm{a})+\alpha_{2} \mathrm{D}^{\mathrm{p}}(\mathrm{fx}, \mathrm{Ty}, \mathrm{a})+\alpha_{3} \mathrm{D}^{\mathrm{p}}(\mathrm{gy}, \mathrm{Sx}, \mathrm{a})$ $+\alpha_{4} \mathrm{D}^{\mathrm{p}}(\mathrm{gy}, \mathrm{Ty}, \mathrm{a})+\alpha_{5} \mathrm{D}^{\mathrm{p}}(\mathrm{fx}, \mathrm{Sx}, \mathrm{a})$ and the result follows from Theorem 3.2.

Corollary 3.4. Let $(X, d)$ be a 2-metric space. $f, g: X \rightarrow X$ and $\mathrm{S}, \mathrm{T}: \mathrm{X} \rightarrow \mathrm{CB}(\mathrm{X})$ satisfying the relation, $\mathrm{D}^{\mathrm{p}}(\mathrm{Sx}, \mathrm{Ty}, \mathrm{a}) \leq$ $\alpha_{1} \mathrm{~d}^{\mathrm{p}}(\mathrm{x}, \mathrm{y}, \mathrm{a})+\alpha_{2} \mathrm{D}^{\mathrm{p}}(\mathrm{x}, \mathrm{Ty}, \mathrm{a})+\alpha_{3} \mathrm{D}^{\mathrm{p}}(\mathrm{y}, \mathrm{Sx}, \mathrm{a})+\alpha_{4} \mathrm{D}^{\mathrm{p}}(\mathrm{y}, \mathrm{Ty}, \mathrm{a})+$ $\alpha_{5} \mathrm{D}^{\mathrm{p}}(\mathrm{x}, \mathrm{Sx}, \mathrm{a})$; where $\mathrm{p} \geq 1, \alpha_{\mathrm{i}} \geq 0, \mathrm{i}=1,2,3,4,5$ and $\sum_{i=1}^{5} \alpha \alpha_{\mathrm{i}}<1$. The pairs (S, f) and (T, g) satisfy OWC-property. $\mathrm{f}(\mathrm{X})$ and $\mathrm{g}(\mathrm{X})$ are closed. Then $\mathrm{f}, \mathrm{g}, \mathrm{S}$ and $\mathrm{T}$ have a unique common fixed point in $\mathrm{X}$.

Proof. Since $\mathrm{D}^{\mathrm{p}}(\mathrm{Sx}, \mathrm{Ty}, \mathrm{a}) \leq \mathrm{H}^{\mathrm{p}}(\mathrm{Sx}, \mathrm{Ty}, \mathrm{a}) \leq \alpha_{1} \mathrm{~d}^{\mathrm{p}}(\mathrm{x}, \mathrm{y}, \mathrm{a})+$ $\alpha_{2} \mathrm{D}^{\mathrm{p}}(\mathrm{x}, \mathrm{Ty}, \mathrm{a})+\alpha_{3} \mathrm{D}^{\mathrm{p}}(\mathrm{y}, \mathrm{Sx}, \mathrm{a})+\alpha_{4} \mathrm{D}^{\mathrm{p}}(\mathrm{y}, \mathrm{Ty}, \mathrm{a})+\alpha_{5} \mathrm{D}^{\mathrm{p}}(\mathrm{x}, \mathrm{Sx}, \mathrm{a})$, the result follows from Colollary 3.2.

Theorem 3.3. Let $(X, d)$ be a 2-metric space. $f, g: X \rightarrow X$ and $\mathrm{S}, \mathrm{T}: \mathrm{X} \rightarrow \mathrm{CB}(\mathrm{X})$ satisfying the relation, $\mathrm{H}^{\mathrm{p}}(\mathrm{Sx}, \mathrm{Ty}, \mathrm{a}) \leq$ $\alpha_{1} \mathrm{~d}^{\mathrm{p}}(\mathrm{fx}, \mathrm{gy}, \mathrm{a})+\alpha_{2} \mathrm{D}^{\mathrm{p}}(\mathrm{fx}, \mathrm{Ty}, \mathrm{a})+\alpha_{3} \mathrm{D}^{\mathrm{p}}(\mathrm{gy}, \mathrm{Sx}, \mathrm{a})+\alpha_{4} \mathrm{D}^{\mathrm{p}}(\mathrm{gy}, \mathrm{Ty}$, a) $+\alpha_{5} \mathrm{D}^{\mathrm{p}}(\mathrm{fx}, \mathrm{Sx}, \mathrm{a}), \mathrm{p} \geq 1, \alpha_{\mathrm{i}} \geq 0, \mathrm{i}=1,2,3,4,5$ and $\sum_{i=1}^{5} \alpha \alpha_{\mathrm{i}}<1$. The pairs (S, f) and (T, g) satisfy CLRf -property. Also (S, f) are weakly compatible, $f$ is continuous, $g$ is one-one and $f(X)$, $\mathrm{g}(\mathrm{X})$ are closed. Then $\mathrm{f}, \mathrm{g}, \mathrm{S}$ and $\mathrm{T}$ have a unique common fixed point in $\mathrm{X}$.

Proof. As the pairs (S, f) and (T, g) satisfy CLRf -property, then there exists two sequences $\left\{x_{n}\right\},\left\{y_{n}\right\}$ in $\mathrm{X}$ and $\mathrm{A}, \mathrm{B} \in \mathrm{CB}(\mathrm{X})$ such that $\lim _{n \rightarrow \infty} \mathrm{S} x_{n}=\mathrm{A} ; \lim _{n \rightarrow \infty} \mathrm{T} y_{n}=\mathrm{B}$ and $\lim _{n \rightarrow \infty} \mathrm{f} x_{n}=\lim _{n \rightarrow \infty} \mathrm{g} y_{n}=$ $\mathrm{fu} \in \mathrm{A} \cap \mathrm{B}$ for some $\mathrm{u} \in \mathrm{X}$. Since $\mathrm{f}(\mathrm{X}), \mathrm{g}(\mathrm{X})$ are closed subsets of $X$, there exist $v, w \in X$ such that $f u=f v$ and $f u=g w$.

We show that gw $\in$ Tw. If possible let gw $\notin$ Tw. Then by the given condition,

$\mathrm{D}^{\mathrm{p}}\left(\mathrm{f} x_{n^{\prime}}, \mathrm{Tw}, \mathrm{a}\right) \leq \mathrm{H}^{\mathrm{p}}\left(\mathrm{S} x_{n^{\prime}}, \mathrm{Tw}, \mathrm{a}\right) \leq \alpha_{1} \mathrm{~d}^{\mathrm{p}}\left(\mathrm{f} x_{n^{\prime}}, \mathrm{gw}, \mathrm{a}\right)+\alpha_{2} \mathrm{D}^{\mathrm{p}}\left(\mathrm{f} x_{n^{\prime}}, \mathrm{Tw}, \mathrm{a}\right)$ $+\alpha_{3} \mathrm{D}^{\mathrm{p}}\left(\mathrm{gw}, \mathrm{S} x_{n^{\prime}} \mathrm{a}\right)+\alpha_{4} \mathrm{D}^{\mathrm{p}}(\mathrm{gw}, \mathrm{Tw}, \mathrm{a})+\alpha_{5} \mathrm{D}^{\mathrm{p}}\left(\mathrm{f} x_{n^{\prime}} \mathrm{S} x_{n^{\prime}} \mathrm{a}\right)$.

Taking $\lim _{n \rightarrow \infty}$ in the above inequality we have $\mathrm{D}^{\mathrm{p}}(\mathrm{fu}$, $\mathrm{Tw}, \mathrm{a}) \leq \alpha_{1} \mathrm{~d}^{\mathrm{p}}(\mathrm{fu}, \mathrm{gw}, \mathrm{a})+\alpha_{2} \mathrm{D}^{\mathrm{p}}(\mathrm{fu}, \mathrm{Tw}, \mathrm{a})+\alpha_{3} \mathrm{D}^{\mathrm{p}}(\mathrm{gw}, \mathrm{A}, \mathrm{a})+$ $\alpha_{4} \mathrm{D}^{\mathrm{p}}(\mathrm{gw}, \mathrm{Tw}, \mathrm{a})+\alpha_{5} \mathrm{D}^{\mathrm{p}}(\mathrm{fu}, \mathrm{A}, \mathrm{a}) \leq \alpha_{1} \cdot 0+\alpha_{2} \mathrm{D}^{\mathrm{p}}(\mathrm{fu}, \mathrm{Tw}, \mathrm{a})+$ $\alpha_{3} \mathrm{D}^{\mathrm{p}}(\mathrm{fu}, \mathrm{A}, \mathrm{a})+\alpha_{4} \mathrm{D}^{\mathrm{p}}(\mathrm{gw}, \mathrm{Tw}, \mathrm{a})+\alpha_{5} .0$

Or, $\mathrm{D}^{\mathrm{p}}(\mathrm{gw}, \mathrm{Tw}, \mathrm{a}) \leq \alpha_{2} \mathrm{D}^{\mathrm{p}}(\mathrm{gw}, \mathrm{Tw}, \mathrm{a})+\alpha_{4} \mathrm{D}^{\mathrm{p}}(\mathrm{gw}, \mathrm{Tw}, \mathrm{a})[$ since $\mathrm{fu}=\mathrm{gw}]$ Or,

$\left(1-\alpha_{2}-\alpha_{4}\right) \mathrm{D}^{\mathrm{p}}(\mathrm{gw}, \mathrm{Tw}, \mathrm{a}) \leq 0$, implies, $1-\alpha_{2}-\alpha_{4} \leq 0$, which is a contradiction. 


\section{Common Fixed Point Theorems on Multi-Valued Mappings in 2-Metric}

Therefore $\mathrm{D}^{\mathrm{p}}(\mathrm{gw}, \mathrm{Tw}, \mathrm{a})=0$, i.e., gw $\in \mathrm{Tw}$ as $\mathrm{n} \rightarrow \infty$.

Now, we show that $f v \in$ Sv. Consider fv $\notin$ Sv.

Since, $\mathrm{D}^{\mathrm{p}}\left(\mathrm{Sv}, \mathrm{g} y_{n^{\prime}}\right.$ a $) \leq \mathrm{H}^{\mathrm{p}}\left(\mathrm{Sv}, \mathrm{T} y_{n^{\prime}}\right.$ a $) \leq \alpha_{1} \mathrm{~d}^{\mathrm{p}}\left(\mathrm{fv}, \mathrm{g} y_{n^{\prime}}\right.$ a $)+$ $\alpha_{2} \mathrm{D}^{\mathrm{p}}\left(\mathrm{fv}, \mathrm{T} y_{n^{\prime}}, \mathrm{a}\right)+\alpha_{3} \mathrm{D}^{\mathrm{p}}\left(\mathrm{g} y_{n^{\prime}}, \mathrm{Sv}, \mathrm{a}\right)+\alpha_{4} \mathrm{D}^{\mathrm{p}}\left(\mathrm{g} y_{n^{\prime}}, \mathrm{T} y_{n^{\prime}}, \mathrm{a}\right)+\alpha_{5} \mathrm{D}^{\mathrm{p}}(\mathrm{fv}, \mathrm{Sv}, \mathrm{a})$.

Taking limit as $\mathrm{n} \rightarrow \infty$ both sides in the above inequality we have $\mathrm{D}^{\mathrm{p}}(\mathrm{Sv}, \mathrm{fu}, \mathrm{a}) \leq \alpha_{1} \mathrm{~d}^{\mathrm{p}}(\mathrm{fv}, \mathrm{fu}, \mathrm{a})+\alpha_{2} \mathrm{D}^{\mathrm{p}}(\mathrm{f}, \mathrm{B}, \mathrm{a})+\alpha_{3} \mathrm{D}^{\mathrm{p}}(\mathrm{fu}, \mathrm{Sv}, \mathrm{a})$ $+\alpha_{4} \mathrm{D}^{\mathrm{p}}(\mathrm{fu}, \mathrm{B}, \mathrm{a})+\alpha_{5} \mathrm{D}^{\mathrm{p}}(\mathrm{fv}, \mathrm{Sv}, \mathrm{a})$ Or, $\mathrm{D}^{\mathrm{p}}(\mathrm{Sv}, \mathrm{fu}, \mathrm{a}) \leq \alpha_{1} \cdot 0+\alpha_{2} .0$ $+\alpha_{3} \mathrm{D}^{\mathrm{p}}(\mathrm{fu}, \mathrm{Sv}, \mathrm{a})+\alpha_{4} \cdot 0+\alpha_{5} \mathrm{~d}^{\mathrm{p}}(\mathrm{fv}, \mathrm{Sv}, \mathrm{a})$

implies, $\left(1-\alpha_{3}-\alpha_{5}\right) \mathrm{D}^{\mathrm{p}}(\mathrm{Sv}, \mathrm{fv}, \mathrm{a}) \leq 0$ i.e., $\mathrm{D}^{\mathrm{p}}(\mathrm{Sv}, \mathrm{fv}, \mathrm{a})=0$ i.e., fv $\in$ Sv.

Now let $v \neq f v$. Since $(S, f)$ is weakly compatible, we have $\mathrm{fv} \in \mathrm{Sv}$ implies, $\mathrm{ffv} \in \mathrm{fSv}=\mathrm{Sfv}$.

Therefore $d^{p}(f f v, f v, a)=D^{p}(f f v, g w, a) \leq H^{p}(S f v, T w, a) \leq$ $\alpha_{1} \mathrm{~d}^{\mathrm{p}}(\mathrm{ffv}, \mathrm{gw}, \mathrm{a})+\alpha_{2} \mathrm{D}^{\mathrm{p}}(\mathrm{ffv}, \mathrm{Tw}, \mathrm{a})+\alpha_{3} \mathrm{D}^{\mathrm{p}}(\mathrm{gw}, \mathrm{Sfv}, \mathrm{a})+\alpha_{4} \mathrm{D}^{\mathrm{p}}(\mathrm{gw}$, $\mathrm{Tw}, \mathrm{a})+\alpha_{5} \mathrm{D}^{\mathrm{p}}(\mathrm{ffv}, \mathrm{Sfv}, \mathrm{a}) \leq \alpha_{1} \mathrm{~d}^{\mathrm{p}}(\mathrm{ffv}, \mathrm{gw}, \mathrm{a})+\alpha_{2} \mathrm{~d}^{\mathrm{p}}(\mathrm{ffv}, \mathrm{gw}, \mathrm{a})+$ $\alpha_{3} \mathrm{~d}^{\mathrm{p}}(\mathrm{fv}, \mathrm{ffv}, \mathrm{a})+\alpha_{4} .0+\alpha_{5} .0=\alpha_{1} \mathrm{~d}^{\mathrm{p}}(\mathrm{ffv}, \mathrm{fv}, \mathrm{a})+\alpha_{2} \mathrm{~d}^{\mathrm{p}}(\mathrm{ffv}, \mathrm{fv}, \mathrm{a})+$ $\alpha_{3} \mathrm{~d}^{\mathrm{p}}(\mathrm{ffv}, \mathrm{fv}, \mathrm{a})$

implies, $\left(1-\alpha_{1}-\alpha_{2}-\alpha_{3}\right) \mathrm{d}^{\mathrm{p}}(\mathrm{ffv}, \mathrm{fv}, \mathrm{a}) \leq 0$ i.e., $\mathrm{d}^{\mathrm{p}}(\mathrm{ffv}, \mathrm{fv}, \mathrm{a})$ $=0$ i.e., $f f v=f v$. i.e., $f v=v$ [since $f$ is continuous].

Again, $d^{p}(v, g v, a)=D^{p}(f v, g v, a) \leq H^{p}(S v, T v, a) \leq$ $\alpha_{1} \mathrm{~d}^{\mathrm{p}}(\mathrm{fv}, \mathrm{gv}, \mathrm{a})+\alpha_{2} \mathrm{D}^{\mathrm{p}}(\mathrm{fv}, \mathrm{Tv}, \mathrm{a})+\alpha_{3} \mathrm{D}^{\mathrm{p}}(\mathrm{gv}, \mathrm{Sv}, \mathrm{a})+\alpha_{4} \mathrm{D}^{\mathrm{p}}(\mathrm{gv}, \mathrm{Tv}, \mathrm{a})$ $+\alpha_{5} \mathrm{D}^{\mathrm{p}}(\mathrm{fv}, \mathrm{Sv}, \mathrm{a}) \leq \alpha_{1} \mathrm{~d}^{\mathrm{p}}(\mathrm{v}, \mathrm{gv}, \mathrm{a})+\alpha_{2} \mathrm{~d}^{\mathrm{p}}(\mathrm{v}, \mathrm{Tv}, \mathrm{a})+\alpha_{3} \mathrm{D}^{\mathrm{p}}(\mathrm{v}, \mathrm{gv}, \mathrm{a})$ $+\alpha_{4} .0+\alpha_{5} .0$ implies, $\left(1-\alpha_{1}-\alpha_{2}-\alpha_{3}\right) \mathrm{d}^{\mathrm{p}}(\mathrm{v}, \mathrm{gv}, \mathrm{a}) \leq 0$ i.e., $\mathrm{d}^{\mathrm{p}}(\mathrm{v}, \mathrm{gv}, \mathrm{a})=0$ i.e., $\mathrm{v}=\mathrm{gv}$.

Therefore, $v=f v=g v$ and $f v \in S v ; g v=f v=f u=g w \in T w$ implies, $g v=g w$ i.e., $v=w$ [since $g$ is one one]. Thus $v=f v=g v$, $f v \in S v$, gv $\in$ Tv.

Thus $v$ is a common fixed point of $f, g, S$ and T. Let $x$ be another common fixed point of $\mathrm{f}, \mathrm{g}, \mathrm{S}$ and $\mathrm{T}$. Then $\mathrm{d}^{\mathrm{p}}(\mathrm{v}, \mathrm{x}, \mathrm{a})=$ $\mathrm{D}^{\mathrm{p}}(\mathrm{fv}, \mathrm{gx}, \mathrm{a}) \leq \mathrm{H}^{\mathrm{p}}(\mathrm{Sv}, \mathrm{Tx}, \mathrm{a}) \leq \alpha_{1} \mathrm{~d}^{\mathrm{p}}(\mathrm{fv}, \mathrm{gx}, \mathrm{a})+\alpha_{2} \mathrm{D}^{\mathrm{p}}(\mathrm{fv}, \mathrm{Tx}, \mathrm{a})+$ $\alpha_{3} \mathrm{D}^{\mathrm{p}}(\mathrm{gx}, \mathrm{Sv}, \mathrm{a})+\alpha_{4} \mathrm{D}^{\mathrm{p}}(\mathrm{gx}, \mathrm{Tx}, \mathrm{a})+\alpha_{5} \mathrm{D}^{\mathrm{p}}(\mathrm{fv}, \mathrm{Sv}, \mathrm{a}) \leq \alpha_{1} \mathrm{~d}^{\mathrm{p}}(\mathrm{v}, \mathrm{x}, \mathrm{a})$ $+\alpha_{2} \mathrm{~d}^{\mathrm{p}}(\mathrm{fv}, \mathrm{gx}, \mathrm{a})+\alpha_{3} \mathrm{~d}^{\mathrm{p}}(\mathrm{gx}, \mathrm{fv}, \mathrm{a})+\alpha_{4} .0+\alpha_{5} .0=\alpha_{1} \mathrm{~d}^{\mathrm{p}}(\mathrm{v}, \mathrm{x}, \mathrm{a})+$ $\alpha_{2} \mathrm{~d}^{\mathrm{p}}(\mathrm{v}, \mathrm{x}, \mathrm{a})+\alpha_{3} \mathrm{~d}^{\mathrm{p}}(\mathrm{x}, \mathrm{v}, \mathrm{a})$

implies, $\left(1-\alpha_{1}-\alpha_{2}-\alpha_{3}\right) \mathrm{d}^{\mathrm{p}}(\mathrm{x}, \mathrm{v}, \mathrm{a}) \leq 0$ i.e., $\mathrm{d}^{\mathrm{p}}(\mathrm{x}, \mathrm{v}, \mathrm{a})=0$ i.e., $\mathrm{x}=\mathrm{v}$. Hence $\mathrm{f}, \mathrm{g}, \mathrm{S}$ and $\mathrm{T}$ has unique common fixed point in $\mathrm{X}$.

Theorem 3.4. Let $(X, d)$ be a 2 -metric space. $f, g: X \rightarrow X$ and $\mathrm{S}, \mathrm{T}: \mathrm{X} \rightarrow \mathrm{CB}(\mathrm{X})$ satisfying the relation, $\mathrm{H}^{\mathrm{p}}(\mathrm{Sx}, \mathrm{Ty}, \mathrm{a}) \leq$ $\alpha_{1} \mathrm{~d}^{\mathrm{p}}(\mathrm{fx}, \mathrm{gy}, \mathrm{a})+\alpha_{2} \mathrm{D}^{\mathrm{p}}(\mathrm{fx}, \mathrm{Ty}, \mathrm{a})+\alpha_{3} \mathrm{D}^{\mathrm{p}}(\mathrm{gy}, \mathrm{Sx}, \mathrm{a})+\alpha_{4} \mathrm{D}^{\mathrm{p}}(\mathrm{gy}, \mathrm{Ty}$, a) $+\alpha_{5} \mathrm{D}^{\mathrm{p}}(\mathrm{fx}, \mathrm{Sx}, \mathrm{a}), \mathrm{p} \geq 1, \alpha_{\mathrm{i}} \geq 0, \mathrm{i}=1,2,3,4,5$ and $\sum_{i=1}^{5} \alpha \alpha_{\mathrm{i}}<1$.

The pairs (S, f) and (T, g) satisfy D-mapping and $f(X)$, $\mathrm{g}(\mathrm{X})$ are closed. Then $\mathrm{f}, \mathrm{g}, \mathrm{S}$ and $\mathrm{T}$ has unique common fixed point in X.
Proof. Let $x_{0} \in \mathrm{X}$ be arbitrary. We construct two sequences $\left\{\mathrm{x}_{\mathrm{n}}\right\}$ and $\left\{\mathrm{y}_{\mathrm{n}}\right\}$ such that $\mathrm{y}_{\mathrm{n}-1}=\mathrm{fx}_{\mathrm{n}} \in \mathrm{Sx}_{\mathrm{n}-1} ; \mathrm{y}_{\mathrm{n}}=$ $\mathrm{gx}_{\mathrm{n}+1} \in \mathrm{Tx}_{\mathrm{n}}$ for all $\mathrm{n} \in \mathrm{N}$.

Now since (S, f) and (T, f) satisfy D-mapping, then for the sequences $\left\{x_{n}\right\}$ and $\left\{y_{n}\right\}$ we have $\lim _{n \rightarrow \infty} \mathrm{fx}_{\mathrm{n}}=\mathrm{x}$ and $\lim _{n \rightarrow \infty} \mathrm{Sx}=$ $\{\mathrm{x}\}$ and $\lim _{n \rightarrow \infty} \mathrm{gy}_{\mathrm{n}}=\mathrm{y}$ and $\lim _{n \rightarrow \infty} \mathrm{Ty}_{\mathrm{n}}=\{\mathrm{y}\}$ for some $\mathrm{x}, \mathrm{y} \in \mathrm{X}$.

Since $\mathrm{D}^{\mathrm{p}}\left(\mathrm{fx}, \mathrm{T} y_{n^{\prime}} \mathrm{a}\right) \leq \mathrm{D}^{\mathrm{p}}\left(\mathrm{fx}, \mathrm{g} y_{n^{\prime}}\right.$ a) $\leq \mathrm{H}^{\mathrm{p}}\left(\mathrm{Sx}, \mathrm{T} y_{n^{\prime}}\right.$ a $) \leq$ $\alpha_{1} \mathrm{~d}^{\mathrm{p}}\left(\mathrm{fx}, \mathrm{g} y_{n^{\prime}}, \mathrm{a}\right)+\alpha_{2} \mathrm{D}^{\mathrm{p}}\left(\mathrm{fx}, \mathrm{T} y_{n^{\prime}} \mathrm{a}\right)+\alpha_{3} \mathrm{D}^{\mathrm{p}}\left(\mathrm{g} y_{n^{\prime}} \mathrm{Sx}, \mathrm{a}\right)+\alpha_{4} \mathrm{D}^{\mathrm{p}}\left(\mathrm{g} y_{n^{\prime}}\right.$ $\mathrm{T} y_{n^{\prime}}$ a $)+\alpha_{5} \mathrm{D}^{\mathrm{p}}(\mathrm{fx}, \mathrm{Sx}, \mathrm{a}) \leq \alpha_{1} \mathrm{~d}^{\mathrm{p}}\left(\mathrm{fx}, \mathrm{g} y_{n^{\prime}} \mathrm{a}\right)+\alpha_{2} \mathrm{D}^{\mathrm{p}}\left(\mathrm{fx}, \mathrm{T} y_{n^{\prime}} \mathrm{a}\right)+$ $\alpha_{3}\left[\mathrm{D}^{\mathrm{p}}\left(\mathrm{g} y_{n^{\prime}}, \mathrm{Sx}, \mathrm{fx}\right)+\mathrm{D}^{\mathrm{p}}\left(\mathrm{g} y_{n^{\prime}}, \mathrm{fx}, \mathrm{a}\right)+\mathrm{D}^{\mathrm{p}}(\mathrm{fx}, \mathrm{Sx}, \mathrm{a})\right]+\alpha_{4} \cdot 0+\alpha_{5} \cdot 0=$ $\alpha_{1} \mathrm{~d}^{\mathrm{p}}\left(\mathrm{fx}, \mathrm{g} y_{n^{\prime}}, \mathrm{a}\right)+\alpha_{2} \mathrm{D}_{\mathrm{p}}\left(\mathrm{fx}, \mathrm{T} y_{n^{\prime}}, \mathrm{a}\right)+\alpha_{3} \mathrm{D}^{\mathrm{p}}\left(\mathrm{fx}, \mathrm{g} y_{n^{\prime}}, \mathrm{a}\right)$

Or, $\left(1-\alpha_{2}-\alpha_{3}\right) \mathrm{D}^{\mathrm{p}}\left(\mathrm{fx}, \mathrm{g} y_{n^{\prime}} \mathrm{a}\right) \leq \alpha_{1} \mathrm{~d}^{\mathrm{p}}\left(\mathrm{fx}, \mathrm{g} y_{n^{\prime}}\right.$ a) Or, $\left(1-\alpha_{2}-\alpha_{3}\right) \mathrm{d}^{\mathrm{p}}\left(\mathrm{fx}, \mathrm{g} y_{n^{\prime}}\right.$ a $) \leq \alpha_{1} \mathrm{~d}^{\mathrm{p}}\left(\mathrm{fx} ; \mathrm{g} y_{n^{\prime}}\right.$ a) Or, $\left(1-\alpha_{1}-\alpha_{2}-\alpha_{3}\right)$ $\mathrm{D}^{\mathrm{p}}\left(\mathrm{fx}, \mathrm{g} y_{n^{\prime}}, \mathrm{a}\right) \leq 0$ implies, $\mathrm{D}^{\mathrm{p}}\left(\mathrm{fx}, \mathrm{g} y_{n}, \mathrm{a}\right)=0$ i.e., $\mathrm{fx}=\mathrm{g} y_{n}$.

i.e., $\lim _{n \rightarrow \infty} \mathrm{fx}=\lim _{n \rightarrow \infty} \mathrm{g} y_{n}=\mathrm{y}$ i.e., $\mathrm{fx}=\mathrm{y}$.

Again, $D^{p}(S x, y, a) \leq d^{p}(f x, y, a)=0$ implies, $y \in S x$. Therefore, $y=f x \in S x$. Also $y \in T y_{n}$.

Now we show that $y=$ gy $\in$ Ty.

Since $D^{p}(y, T y, a)=D^{p}(f x, T y, a) \leq H^{p}(S x, T y, a) \leq \alpha_{1} d^{p}(f x$, gy, a) $+\alpha_{2} \mathrm{D}^{\mathrm{p}}(\mathrm{fx}, \mathrm{Ty}, \mathrm{a})+\alpha_{3} \mathrm{D}^{\mathrm{p}}(\mathrm{gy}, \mathrm{Sx}, \mathrm{a})+\alpha_{4} \mathrm{D}^{\mathrm{p}}(\mathrm{gy}, \mathrm{Ty}, \mathrm{a})+$ $\alpha_{5} \mathrm{D}^{\mathrm{p}}(\mathrm{fx}, \mathrm{Sx}, \mathrm{a}) \leq \alpha_{1} \mathrm{~d}^{\mathrm{p}}(\mathrm{fx}, \mathrm{gy}, \mathrm{a})+\alpha_{2} \mathrm{D}^{\mathrm{p}}(\mathrm{y}, \mathrm{Ty}, \mathrm{a})+\alpha_{3} \mathrm{D}^{\mathrm{p}}(\mathrm{gy}, \mathrm{fx}$, a) $+\alpha_{4^{\cdot}} .0+\alpha_{5} .0$ implies, $\left(1-\alpha_{2}\right) \mathrm{D}^{\mathrm{p}}(\mathrm{y}, \mathrm{Ty}, \mathrm{a}) \leq \alpha_{1} \mathrm{~d}^{\mathrm{p}}(\mathrm{y}, \mathrm{gy}, \mathrm{a})+$ $\alpha_{3} \mathrm{D}^{\mathrm{p}}(\mathrm{gy}, \mathrm{y}, \mathrm{a})$ i.e., $\left(1-\alpha_{2}\right) \mathrm{D}^{\mathrm{p}}(\mathrm{y}, \mathrm{Ty}, \mathrm{a}) \leq\left(\alpha_{1}+\alpha_{3}\right) \mathrm{D}^{\mathrm{p}}(\mathrm{y}, \mathrm{gy}, \mathrm{a})$.

Let $D^{p}(y, T y, a) \neq D^{p}(y, g y, a)$. Then from the above inequality we get

$$
\left(\frac{1-\alpha_{2}}{\alpha_{1}+\alpha_{3}}\right) D^{p}(y, T y, a) \leq D^{p}(y, g y, a)
$$

Since $D^{p}(y, T y, a) \leq D^{p}(y, g y, a)$ using (2.5), we have $\frac{1-\alpha_{2}}{\alpha_{1}+\alpha_{3}} \leq 1$ implies, $1-\alpha_{2} \leq \alpha_{1}+\alpha_{3}$

i.e., $1 \leq \alpha_{1}+\alpha_{2}+\alpha_{3}$, which is a contradiction.

Therefore $D^{p}(y, T y, a)=D^{p}(y, g y, a)=d^{p}(y, g y, a)$. From (2.5) we have

$$
\left(\frac{1-\alpha_{2}}{\alpha_{1}+\alpha_{3}}\right) d^{\mathrm{p}}(\mathrm{y}, \mathrm{gy}, \mathrm{a}) \leq \mathrm{d}^{\mathrm{p}}(\mathrm{y}, \mathrm{gy}, \mathrm{a}) \text { implies, }\left(\frac{1-\alpha_{2}-\alpha_{1}-\alpha_{3}}{\alpha_{1}+\alpha_{3}}\right)
$$

$d^{p}(y, g y, a) \leq 0$ i.e., $d^{p}(y, g y, a)=0$ i.e., $y=$ gy $\in$ Ty.

Next we show that $\mathrm{x}=\mathrm{y}$.

So, $\lim _{n \rightarrow \infty} \mathrm{D}^{\mathrm{p}}(\mathrm{x}, \mathrm{y}, \mathrm{a}) \leq \lim _{n \rightarrow \infty} \mathrm{H}^{\mathrm{p}}\left(\mathrm{S} x_{n^{\prime}} \mathrm{T} y_{n^{\prime}} \mathrm{a}\right) \leq \lim _{n \rightarrow \infty}\left[\alpha_{1} \mathrm{~d}^{\mathrm{p}}\left(\mathrm{f} x_{n^{\prime}}, \mathrm{g} y_{n^{\prime}} \mathrm{a}\right)+\right.$ $\alpha_{2} \mathrm{D}^{\mathrm{p}}\left(\mathrm{f} x_{n^{\prime}}, \mathrm{T} y_{n^{\prime}}, \mathrm{a}\right)+{ }^{n} \alpha_{3} \mathrm{D}^{\mathrm{p}}\left(\mathrm{g} y_{n^{\prime}} \mathrm{S} x_{n^{\prime}}, \mathrm{a}\right)+\alpha_{4} \mathrm{D}^{\mathrm{p}}\left(\mathrm{g} y_{n^{\prime}}, \mathrm{T} y_{n^{\prime}}\right.$ a) + $\left.\alpha_{5} \mathrm{D}^{\mathrm{p}}\left(\mathrm{f} x_{n}, \mathrm{~S} x_{n}, \mathrm{a}\right)\right]$

$=\alpha_{1} \mathrm{~d}^{\mathrm{p}}(\mathrm{x}, \mathrm{y}, \mathrm{a})+\alpha_{2} \mathrm{D}^{\mathrm{p}}(\mathrm{x},\{\mathrm{y}\}, \mathrm{a})+\alpha_{3} \mathrm{D}^{\mathrm{p}}(\mathrm{y},\{\mathrm{x}\}, \mathrm{a})+\alpha_{4} \cdot 0+\alpha_{5} \cdot 0$ 
implies, $\mathrm{d}^{\mathrm{p}}(\mathrm{x}, \mathrm{y}, \mathrm{a}) \leq\left(\alpha_{1}+\alpha_{2}+\alpha_{3}\right) \mathrm{d}^{\mathrm{p}}(\mathrm{x}, \mathrm{y}$, a $)$ i.e., $\mathrm{d}^{\mathrm{p}}(\mathrm{x}, \mathrm{y}, \mathrm{a})=0$ i.e., $x=y$.

Therefore, $x=y=f x \in S x ; x=y=g x \in T x$.

$$
\text { Also, } \lim _{n \rightarrow \infty} S x_{n}=\{x\}=\{y\}=\lim _{n \rightarrow \infty} T y_{n} \text {. }
$$

Again, $\mathrm{D}^{\mathrm{p}}(\mathrm{Sx}, \mathrm{Ty}, \mathrm{a}) \leq \mathrm{H}^{\mathrm{p}}(\mathrm{Sx}, \mathrm{Ty}, \mathrm{a}) \leq \alpha_{1} \mathrm{~d}^{\mathrm{p}}(\mathrm{fx}, \mathrm{gy}, \mathrm{a})+$ $\left.\alpha_{2} \mathrm{D}^{\mathrm{p}}(\mathrm{fx}, \mathrm{Tx}, \mathrm{a})+\alpha_{3} \mathrm{D}^{\mathrm{p}}(\mathrm{gx}, \mathrm{Sx}, \mathrm{a})+\alpha_{4} \mathrm{D}^{\mathrm{p}}(\mathrm{gy}, \mathrm{Tx}, \mathrm{a})+\alpha_{5} \mathrm{D}^{\mathrm{p}}(\mathrm{fx}, \mathrm{Sx}, \mathrm{a})\right]$ $=\alpha_{1} \cdot 0+\alpha_{2} \cdot 0+\alpha_{3} \cdot 0+\alpha_{4} \cdot 0+\alpha_{5} \cdot 0=0$ implies, $\mathrm{Sx}=\mathrm{Ty}=\{\mathrm{x}\}$.

Thus $\mathrm{x}$ is common fixed point of $\mathrm{f}, \mathrm{g}, \mathrm{S}$ and $\mathrm{T}$. To show the uniqueness of $\mathrm{x}$, let $\mathrm{z}$ be another fixed point.

Then $\mathrm{D}^{\mathrm{p}}(\mathrm{x}, \mathrm{z}, \mathrm{a}) \leq \mathrm{H}^{\mathrm{p}}(\mathrm{Sx}, \mathrm{Tz}, \mathrm{a}) \leq \alpha_{1} \mathrm{~d}^{\mathrm{p}}(\mathrm{fx}, \mathrm{gz}, \mathrm{a})+\alpha_{2} \mathrm{D}^{\mathrm{p}}(\mathrm{fx}, \mathrm{Tz}, \mathrm{a})$ $+\alpha_{3} \mathrm{D}^{\mathrm{p}}(\mathrm{gz}, \mathrm{Sx}, \mathrm{a})+\alpha_{4} \mathrm{D}^{\mathrm{p}}(\mathrm{gz}, \mathrm{Tz}, \mathrm{a})+\alpha_{5} \mathrm{D}^{\mathrm{p}}(\mathrm{fx}, \mathrm{Sx}, \mathrm{a})$

$=\alpha_{1} \mathrm{~d}^{\mathrm{p}}(\mathrm{x}, \mathrm{z}, \mathrm{a})+\alpha_{2} \mathrm{D}^{\mathrm{p}}(\mathrm{x}, \mathrm{Tz}, \mathrm{a})+\alpha_{3} \mathrm{D}^{\mathrm{p}}(\mathrm{z}, \mathrm{Sx}, \mathrm{a})+\alpha_{4} \cdot 0+\alpha_{5^{\circ}} 0$

$\leq\left(\alpha_{1}+\alpha_{2}+\alpha_{3}\right) \mathrm{D}^{\mathrm{p}}(\mathrm{x}, \mathrm{z}, \mathrm{a})$

If $D^{p}(x, z, a) \neq d^{p}(x, z$ a $)$, then since $D^{p}(x, z, a) \leq d^{p}(x, z a)$, we have from (2.6), $1 \leq \alpha_{1}+\alpha_{2}+\alpha_{3}$, which is a contradiction by the given condition. Therefore $\mathrm{D}^{\mathrm{p}}(\mathrm{x}, \mathrm{z}, \mathrm{a})=\mathrm{d}^{\mathrm{p}}(\mathrm{x}, \mathrm{z}$ a)

implies, $\mathrm{x}=\mathrm{z}$. Hence $\mathrm{f}, \mathrm{g}, \mathrm{S}$ and $\mathrm{T}$ have unique common fixed point in $X$.

Wardowski (2012) has defined the set $\mathbf{F}$ of all functions F : $\mathbf{R}+\rightarrow \mathbf{R}$ satisfying the conditions:

\section{I): $F$ is strictly increasing;}

II): for all sequence $\left\{\alpha_{n}\right\}$ in $R+, \lim _{n \rightarrow \infty} \alpha_{n}=0$ if and only if $\lim _{n \rightarrow \infty} F\left(\alpha_{n}\right)=-\infty ;$

III): there exists $0<\mathrm{k}<1$ such that $\lim _{\alpha \rightarrow 0_{+}} \alpha^{k} F(\alpha)=0$.

Theorem 3.5. Let $(X, d)$ be a complete 2-metric space and let $\mathrm{T}: \mathrm{X} \rightarrow \mathrm{CB}(\mathrm{X})$ be a multivalued mapping. Also let $\mathrm{F} \in$ $F$ is a function continuous from right and for $\tau \in R+$ such that $2 \tau+\mathrm{F}(\mathrm{H}(\mathrm{Tx}, \mathrm{Ty}, \mathrm{a})) \leq \mathrm{F}\left(\alpha_{1} \mathrm{~d}(\mathrm{x}, \mathrm{y}, \mathrm{a})+\alpha_{2} \mathrm{D}(\mathrm{x}, \mathrm{Tx}, \mathrm{a})+\alpha_{3} \mathrm{D}(\mathrm{y}, \mathrm{Ty}\right.$, a) $+\alpha_{4} \mathrm{D}(\mathrm{x}, \mathrm{Ty}, \mathrm{a})+\alpha_{5} \mathrm{D}(\mathrm{y}, \mathrm{Tx}, \mathrm{a})$ ) for all $\mathrm{x}, \mathrm{y} \in \mathrm{X}$ where $\alpha_{\mathrm{i}} \geq 0$; $\mathrm{i}=1,2,3,4,5$ and $\alpha_{1}+\alpha_{2}+\alpha_{3}+3 \alpha_{4}+\alpha_{5}<1$.

Then $\mathrm{T}$ has a unique fixed point in $\mathrm{X}$.

Proof. Let for arbitrary $x_{0} \in \mathrm{X}$ we choose a point $x_{1} \in \mathrm{X}$ such that $x_{1} \in \mathrm{T} x_{0}$. If $x_{1} \in \mathrm{T} x_{1}$ then $x_{1}$ is a fixed point of $\mathrm{T}$ and the proof completed. Clearly $\mathrm{D}\left(x_{0}, \mathrm{~T} x_{0}, \mathrm{a}\right) \leq \mathrm{d}\left(x_{0}, x_{1}, \mathrm{a}\right)$.

Therefore, $2 \tau+\mathrm{F}\left(\mathrm{H}\left(\mathrm{T} x_{0}, \mathrm{~T} x_{1}, \mathrm{a}\right)\right) \leq \mathrm{F}\left(\alpha_{1} \mathrm{~d}\left(x_{0}, x_{1}, \mathrm{a}\right)+\right.$ $\alpha_{2} \mathrm{D}\left(x_{0}, \mathrm{~T} x_{0}, \mathrm{a}\right)+\alpha_{3} \mathrm{D}\left(x_{1}, \mathrm{~T} x_{1}, \mathrm{a}\right)+\alpha_{4} \mathrm{D}\left(x_{0}, \mathrm{~T} x_{1}, \mathrm{a}\right)+$ $\left.\alpha_{5} \mathrm{D}\left(x_{1}, \mathrm{~T} x_{0}, \mathrm{a}\right)\right) \leq \mathrm{F}\left(\alpha_{1} \mathrm{~d}\left(x_{0}, x_{1}, \mathrm{a}\right)+\alpha_{2} \mathrm{~d}\left(x_{0}, x_{1}, \mathrm{a}\right)+\right.$ $\left.\alpha_{3} \mathrm{D}\left(x_{1}, \mathrm{~T} x_{1}, \mathrm{a}\right)+\alpha_{4} \mathrm{D}\left(x_{0}, \mathrm{~T} x_{1}, \mathrm{a}\right)+\alpha_{5} .0\right)$

Since $(\mathrm{X}, \mathrm{d})$ is a complete 2-metric space, we have for 0 $<\alpha<1, \mathrm{H}\left(\mathrm{T} x_{0}, \mathrm{~T} x_{1}, \mathrm{a}\right) \leq \alpha \mathrm{d}\left(x_{0}, x_{1}, \mathrm{a}\right)$.
Let us choose $h>1$ such that $d(p, q, a) \leq H\left(T_{p}, T_{q}, a\right)$ where $\mathrm{p} \in \mathrm{Tp}, \mathrm{q} \in \mathrm{Tq}$. Thus there exists $x_{2} \in \mathrm{T} x_{1}$ such that $\mathrm{D}\left(x_{1}, \mathrm{~T} x_{1}, \mathrm{a}\right) \leq \mathrm{d}\left(x_{1}, x_{2}, \mathrm{a}\right) \leq \mathrm{h} \mathrm{H}\left(\mathrm{T} x_{0}, \mathrm{~T} x_{1}, \mathrm{a}\right)$.

Thus in general $\mathrm{d}\left(x_{n}, x_{m}, \mathrm{a}\right) \leq \mathrm{hH}\left(\mathrm{Tx}_{\mathrm{n}-1}, \mathrm{Tx}_{\mathrm{m}-1}, \mathrm{a}\right)$; where $x_{n} \in \mathrm{Tx}_{\mathrm{n}-1}$ for all $\mathrm{n} \in \mathrm{N}$.

Since $\mathrm{F}$ is continuous from right, for $\mathrm{h}>1$ we have from (2.7) and (2.8)

$\mathrm{F}\left(\mathrm{d}\left(x_{1}, x_{2}, \mathrm{a}\right)\right) \leq \mathrm{F}\left(\mathrm{hH}\left(\mathrm{T} x_{0}, \mathrm{~T} x_{1}, \mathrm{a}\right)\right) \leq \mathrm{H}\left(\mathrm{T} x_{0}, \mathrm{~T} x_{1}, \mathrm{a}\right)+\tau$

implies, $2 \tau+\mathrm{F}\left(\mathrm{d}\left(x_{1}, x_{2}, \mathrm{a}\right)\right) \leq 2 \tau+\mathrm{F}\left(\mathrm{H}\left(\mathrm{T} x_{0}, \mathrm{~T} x_{1}, \mathrm{a}\right)\right)+\tau$ $\leq \mathrm{F}\left(\alpha_{1} \mathrm{~d}\left(x_{0}, x_{1}, \mathrm{a}\right)+\alpha_{2} \mathrm{D}\left(x_{0}, \mathrm{~T} x_{0}, \mathrm{a}\right)+\alpha_{3} \mathrm{D}\left(x_{1}, \mathrm{~T} x_{1}, \mathrm{a}\right)+\right.$ $\left.\alpha_{4} \mathrm{D}\left(x_{0}, \mathrm{~T} x_{1}, \mathrm{a}\right)\right)+\tau$

implies, $\tau+\mathrm{F}\left(\mathrm{d}\left(x_{1}, x_{2}, \mathrm{a}\right)\right) \leq \mathrm{F}\left(\left(\alpha_{1}+\alpha_{2}\right) \mathrm{d}\left(x_{0}, x_{1}, \mathrm{a}\right)+\right.$ $\left.\alpha_{3} \mathrm{~d}\left(x_{1}, x_{2}, \mathrm{a}\right)+\alpha_{4}\left(\mathrm{~d}\left(x_{0}, x_{2}, x_{1}\right)+\mathrm{d}\left(x_{0}, x_{1}, \mathrm{a}\right)+\mathrm{d}\left(x_{1}, x_{2}, \mathrm{a}\right)\right)\right)$

i.e., $\mathrm{d}\left(x_{1}, x_{2}, \mathrm{a}\right) \leq\left(\alpha_{1}+\alpha_{2}+2 \alpha_{4}\right) \mathrm{d}\left(x_{0}, x_{1}, \mathrm{a}\right)+\left(\alpha_{3}+\alpha_{4}\right) \mathrm{d}\left(x_{1}, x_{2}, \mathrm{a}\right)$

i.e., $\left(1-\alpha_{3}-\alpha_{4}\right) \mathrm{d}\left(x_{1}, x_{2}, \mathrm{a}\right) \leq\left(\alpha_{1}+\alpha_{2}+2 \alpha 4\right) \mathrm{d}\left(x_{0}, x_{1}, \mathrm{a}\right)$

i.e., $\mathrm{d}\left(x_{1}, x_{2}, \mathrm{a}\right) \leq\left(\frac{\alpha_{1}+\alpha_{2}+2 \alpha_{4}}{1-\alpha_{3}-\alpha_{4}}\right) \mathrm{d}\left(x_{0}, x_{1}\right.$, a $)<\mathrm{d}\left(x_{0}, x_{1}\right.$, a)

[since $\left.\frac{\alpha_{1}+\alpha_{2}+2 \alpha_{4}}{1-\alpha_{3}-\alpha_{4}}<1\right]$.

Therefore, $\tau+\mathrm{F}\left(\mathrm{d}\left(x_{1}, x_{2}, \mathrm{a}\right)\right) \leq \mathrm{F}\left(\mathrm{d}\left(x_{0}, x_{1}, \mathrm{a}\right)\right)$.

Continuing this process we can get a sequence $\left\{x_{n}\right\}$ $\in \mathrm{X}$ such that $x_{n} \notin \mathrm{T} x_{n}, x_{n+1} \in \mathrm{T} x_{n}$ and $\tau+\mathrm{F}\left(\mathrm{d}\left(x_{\mathrm{n}-1}, x_{n}, \mathrm{a}\right)\right) \leq$ $\mathrm{F}\left(\mathrm{d}\left(\mathrm{x}_{\mathrm{n}-2}, \mathrm{x}_{\mathrm{n}-1}, \mathrm{a}\right)\right) \forall \in \mathbf{N}$.

Therefore, $\mathrm{F}\left(\mathrm{d}\left(x_{n}, x_{n+1}, \mathrm{a}\right)\right) \leq \mathrm{F}\left(\mathrm{d}\left(x_{n-1}, x_{n}, \mathrm{a}\right)\right)-\tau \leq \mathrm{F}\left(\mathrm{d}\left(x_{n-2}\right.\right.$, $\left.\left.x_{n-1}, \mathrm{a}\right)\right)-2 \tau \leq \ldots \leq \mathrm{F}\left(\mathrm{d}\left(x_{0}, x_{1}, \mathrm{a}\right)\right)-\mathrm{n} \tau$.

Since $\mathrm{F} \in \mathbf{F}$, taking limit as $\mathrm{n} \rightarrow \infty$,

we have $\lim _{n \rightarrow \infty} \mathrm{F}\left(\mathrm{d}\left(x_{n}, x_{n+1}, \mathrm{a}\right)\right)=-\infty$ if $\mathrm{f} \lim _{n \rightarrow \infty} \mathrm{d}\left(x_{n}, x_{n+1}, \mathrm{a}\right)=0$ and so $\lim _{n \rightarrow \infty} \mathrm{d}\left(x_{n^{\prime}}, x_{n+1}, \mathrm{a}\right)=0$.

Let $\mathrm{n}>\mathrm{m}>n_{1} \in \mathrm{N}$. Then $\lim _{n, m \rightarrow \infty} \mathrm{d}\left(x_{n^{\prime}}, x_{m^{\prime}}\right.$ a $) \leq \lim _{n, m \rightarrow \infty} \mathrm{d}\left(x_{n^{\prime}}\right.$ $\left.\left.x_{m^{\prime}}, x_{n-1}\right)+\mathrm{d}\left(x_{n^{\prime}}, x_{n-1}, \mathrm{a}\right)+\mathrm{d}\left(x_{n-1}, x_{m^{\prime}} \mathrm{a}\right)\right]=\lim _{n, m \rightarrow \infty} \mathrm{d}\left(x_{n^{\prime}}, x_{m^{\prime}}, x_{n-1}\right)+$ $\lim _{n, m \rightarrow \infty} \mathrm{d}\left(x_{n^{\prime}}, x_{n-1}, \mathrm{a}\right)+\lim _{n, m \rightarrow \infty} \mathrm{d}\left(x_{n-1}, x_{m}, \mathrm{a}\right)=\lim _{n, m \rightarrow \infty} \mathrm{d}\left(x_{n-1}, x_{m^{\prime}}, \mathrm{a}\right) \leq$ $\mathrm{d}\left(x_{m^{\prime}}, x_{m}, \mathrm{a}\right)=0$.

Thus $\left\{x_{n}\right\}$ is a Cauchy sequence. Since $\mathrm{X}$ is complete, there exists $\mathrm{x} \in \mathrm{X}$ such that $x_{n} \rightarrow \mathrm{x}$, as $\mathrm{n} \rightarrow \infty$. Thus there exists a subsequence $\left\{x_{n_{k}}\right\}$ such that $x_{n_{k}} \in$ Tx for all $n_{k} \in \mathbf{N}$. As T is closed and $x_{n_{k}} \rightarrow \mathrm{x}$. So we get $\mathrm{x} \in \mathrm{Tx}$ and the proof is completed. So we assume that there exists $n_{0} \in \mathrm{N}$ such that $\mathrm{x} \notin \mathrm{Tx}$ for all $\mathrm{n} \geq n_{0} \in \mathbf{N}$. 
$\operatorname{Now} \mathrm{D}\left(x_{n}, \mathrm{Tx}, \mathrm{a}\right) \leq \mathrm{d}\left(x_{n}, x_{n k}, \mathrm{a}\right) \leq \mathrm{hH}\left(\mathrm{Tx}_{\mathrm{n}-1}, \mathrm{Tx}, \mathrm{a}\right)$ [using (2.9)] implies, $F\left(D\left(x_{n}, T x, a\right)\right) \leq F\left(h H\left(T x_{n-1}, T x, a\right)\right) \leq F\left(H\left(T x_{n-1}, T x, a\right)\right)+\tau$ i.e., $2 \tau+F\left(D\left(x_{n}, T x, a\right)\right) \leq 2 \tau+F\left(H\left(T_{n-1}, T x, a\right)\right)+\tau$

$\leq \mathrm{F}\left(\alpha_{1} \mathrm{~d}\left(x_{n-1}, \mathrm{x}, \mathrm{a}\right)+\alpha_{2} \mathrm{D}\left(x_{n-1}, \mathrm{Tx}_{\mathrm{n}-1}, \mathrm{a}\right)+\alpha_{3} \mathrm{D}(\mathrm{x}, \mathrm{Tx}, \mathrm{a})+\right.$ $\left.\alpha_{4} \mathrm{D}\left(x_{n-1}, \mathrm{Tx}, \mathrm{a}\right)+\alpha_{5} \mathrm{D}\left(\mathrm{x}, \mathrm{T} x_{n-1}, \mathrm{a}\right)\right)+\tau$

implies, $\tau+\mathrm{F}\left(\mathrm{D}\left(x_{n^{\prime}}, \mathrm{T}, \mathrm{a}\right)\right) \leq \mathrm{F}\left(\alpha_{1} \mathrm{~d}\left(x_{n-1}, \mathrm{x}, \mathrm{a}\right)+\alpha_{2} \mathrm{D}\left(\mathrm{x}_{\mathrm{n}-1}, \mathrm{Tx}_{\mathrm{n}-1}, \mathrm{a}\right)\right.$ $+\alpha_{3} \mathrm{D}(\mathrm{x}, \mathrm{Tx}, \mathrm{a})+\alpha_{4} \mathrm{D}\left(\mathrm{x}_{\mathrm{n}-1}, \mathrm{Tx}\right.$, a) i.e., $\mathrm{F}\left(\mathrm{D}\left(x_{n^{\prime}} \mathrm{Tx}, \mathrm{a}\right)\right) \leq \tau+$ $\mathrm{F}\left(\mathrm{D}\left(x_{n}, \mathrm{Tx}, \mathrm{a}\right)\right)$

$\leq \mathrm{F}\left(\alpha_{1} \mathrm{~d}\left(\mathrm{x}_{\mathrm{n}-1}, \mathrm{x}, \mathrm{a}\right)+\alpha_{2} \mathrm{D}\left(\mathrm{x}_{\mathrm{n}-1}, \mathrm{Tx} \mathrm{n}-1, \mathrm{a}\right)+\alpha_{3} \mathrm{D}(\mathrm{x}, \mathrm{Tx}, \mathrm{a})+\right.$ $\left.\alpha_{4} \mathrm{D}\left(\mathrm{x}_{\mathrm{n}-1}, \mathrm{Tx}, \mathrm{a}\right)\right)$

implies, $\mathrm{D}\left(x_{n^{\prime}}, \mathrm{Tx}, \mathrm{a}\right) \leq \alpha_{1} \mathrm{~d}\left(\mathrm{x}_{\mathrm{n}-1}, \mathrm{x}, \mathrm{a}\right)+\alpha_{2} \mathrm{D}\left(\mathrm{x}_{\mathrm{n}-1}, \mathrm{Tx}_{\mathrm{n}-1}, \mathrm{a}\right)+$ $\alpha_{3} \mathrm{D}(\mathrm{x}, \mathrm{Tx}, \mathrm{a})+\alpha_{4} \mathrm{D}\left(\mathrm{x}_{\mathrm{n}-1}, \mathrm{Tx}, \mathrm{a}\right)$.

Taking limit as $\mathrm{n} \rightarrow \infty$ we get, $\mathrm{D}(\mathrm{x}, \mathrm{Tx}, \mathrm{a}) \leq \alpha_{1} .0+$ $\alpha_{2} \mathrm{D}(\mathrm{x}, \mathrm{Tx}, \mathrm{a})+\alpha_{3} \mathrm{D}(\mathrm{x}, \mathrm{Tx}, \mathrm{a})+\alpha_{4} \mathrm{D}(\mathrm{x}, \mathrm{Tx}, \mathrm{a})$ implies, $\left(1-\alpha_{2}-\alpha_{3}-\alpha_{4}\right) \mathrm{D}(\mathrm{x}, \mathrm{Tx}, \mathrm{a}) \leq 0$ implies, $\mathrm{D}(\mathrm{x}, \mathrm{Tx}, \mathrm{a})=0$ implies, $\mathrm{x} \in \mathrm{Tx}$.

Therefore, $\mathrm{T}$ has a fixed point. To show the uniqueness, let $y$ be another fixed point of T. Then $d(x, y, a) \leq h H(T x, T y$, a) implies, $F(d(x, y, a)) \leq F(h H(T x, T y, a)) \leq F(H(T x, T y, a))$.

Therefore, $2 \tau+F(d(x, y, a)) \leq 2 \tau+F(H(T x, T y, a))+\tau$ $\leq \mathrm{F}\left(\alpha_{1} \mathrm{~d}(\mathrm{x}, \mathrm{y}, \mathrm{a})+\alpha_{2} \mathrm{D}(\mathrm{x}, \mathrm{Tx}, \mathrm{a})+\alpha_{3} \mathrm{D}(\mathrm{y}, \mathrm{Ty}, \mathrm{a})+\alpha_{4} \mathrm{D}(\mathrm{x}, \mathrm{Ty}, \mathrm{a})\right.$ $\left.+\alpha_{5} \mathrm{D}(\mathrm{y}, \mathrm{Tx}, \mathrm{a})\right)$

implies, $\tau+F(d(x, y, a)) \leq F\left(\alpha_{1} d(x, y, a)+\alpha_{2} D(x, T x, a)+\right.$ $\left.\alpha_{3} \mathrm{D}(\mathrm{y}, \mathrm{Ty}, \mathrm{a})+\alpha_{4} \mathrm{D}(\mathrm{x}, \mathrm{Ty}, \mathrm{a})+\alpha_{5} \mathrm{D}(\mathrm{y}, \mathrm{Tx}, \mathrm{a})\right)$.

So,F $(d(x, y, a)) \leq \tau+F(d(x, y, a)) \leq F\left(\alpha_{1} d(x, y, a)+\right.$ $\left.\alpha_{2} D(x, T x, a)+\alpha_{3} D(y, T y, a)+\alpha_{4} D(x, T y, a)+\alpha_{5} D(y, T x, a)\right)$.

Therefore, $d(x, y, a) \leq \alpha_{1} d(x, y, a)+\alpha_{2} \cdot 0+\alpha_{3} \cdot 0+$ $\alpha_{4} D(x, T y, a)+\alpha_{5} D(y, T x, a)$

implies, $d(x, y, a) \leq \alpha_{1} d(x, y, a)+\alpha_{4} d(x, y, a)+\alpha_{5} D(y, x, a) \leq$ $\alpha_{1} d(x, y, a)+\alpha_{4} d(x, y, a)+\alpha_{5} d(y, x, a)$.

If $d(x, y, a) \neq 0$ then we have $\left(1-\alpha_{1}-\alpha_{4}-\alpha_{5}\right) \leq 0$ which contradicts the given condition.

Therefore $d(x, y, a)=0$ i.e., $x=y$.

\section{CONCLUSION}

We have reformulated the theorem of Nadler (1969) in 2-metric space for set valued mappings and we have also established some theorems which will give scope for the further works in this area.

\section{ACKNOWLEDGEMENT}

The authors are thankful to the referee for the suggestions towards the improvement of the paper.

\section{REFERENCES}

Abbas M, Rhoades BE(2009) Common fixed point theorems for hybrid pairs of occasionally weakly compatible mappings and applications. Navi Sad J. Math., 39: 89 - 109.

Abd El-Monsef ME, Abu-Donia HM, Abd-Rabou K(2007) Common Fixed Point Theorems of Single and Set-Valued Mappings on 2-Metric Spaces. Applied Mathematics \& Information Science An International Journal,Vol.1(2):185-194.

Abdou AAN(2016) Common fixed point result for multi-valued mappings with, some examples. J. Nonlinear Sci. Appl. 9: 787-798.

Ahmad J, Al-Rawashdeh A, Azam A(2015) New fixed point theorems for generalized F-contractions in complete metric spaces. Fixed Point Theory and Application:2015:80.

Banach S(1922) Sur les operations dans les ensembles abstrits et leur applications aux equations integrals. Fund. Math., 3: 133-181.

Bouhadjera H, Djoudi A(2008) Common fixed point theorems for single and set-valued maps without continuity. An. St. Univ. Ovidius Constanta. Vol. 16(1): 49-58.

Cho SH(2016) Fixed Point for Multivalued Mappings in b-Metris Spaces, Applied Mathematical Sciences. Vol.10: no.59: 29272944.

Djoudi A, Khemis R(2005), Fixed points for set and single valued maps without continuity. Demonstratio Mathematica, 38(3): 739-751.

Gahler S(1963) 2-metricsche Raume und ihre topologische structure. Math. Nachr. 26: 115-148.

Iseki K(1975) Fixed point theorems in 2-metric Spaces. Math. Sem. Notes. Kobe. Univ., 3: 133-136.

Jinakul C, Wiwatwanich A, Kaewkhao A(2017) Common Fixed Point Theorem For Multi-Valued Mappings ON BMETRIC SPACES. Int. J. of Pure and Appl.Math., Vol.113: No.1: 167 - 179.

Joseph JM, Ramganesh E(2013) Fixed Point Theorem on MultiValued Mappings. Int. J.of Ana.b and App. Vol.1:no 2: 123-127.

Jungck G(1986) Compatible mappings and common fixed points. Internat. J. Math. Math. Sci., 9: 771 - 779.

Nadler SB Jr(1969) Multi-Valued Contraction Mappings, Pacific Journal of Mathematics. Vol. 30: No. 2: 475-488.

Sintunavarat W, Kumar P(2011) Common fixed point theorems for a pair of weakly compatible mappings in fuzzy metric spaces. Hindawi Publishing Corporation Journal of Applied Mathematics, Volume 2011, Article ID 637958: 1-14.

Wardowski D(2012) Fixed points of a new type of contractive mappings in complete metric spaces. Fixed Point Theory and Applications, 2012:94: 1-6 